\title{
LOOP TOP HARD X-RAY EMISSION IN SOLAR FLARES: IMAGES AND STATISTICS
}

\author{
Vahé Petrosian $^{1}$ and Timothy Q. Donaghy ${ }^{2}$ \\ Center for Space Science and Astrophysics, Stanford University, Stanford, CA 94305-4060 \\ AND \\ JAMES M. MCTIERNAN \\ Space Sciences Laboratory, University of California, Berkeley, CA 94720-7450 \\ Received 2001 May 7; accepted 2001 December 17
}

\begin{abstract}
The discovery of hard X-ray sources near the top of a flaring loop by the Hard X-Ray Telescope on board the Yohkoh satellite represents a significant progress toward the understanding of the basic processes driving solar flares. In this paper we extend the previous study of limb flares by Masuda by including all Yohkoh observations up through 1998 August. We report that from 1991 October to 1998 August, Yohkoh observed $20 \mathrm{X}$-ray-bright limb flares (where we use the same selection criteria as Masuda), of which we have sufficient data to analyze 18 events, including eight previously unanalyzed flares. Of these 18 events, 15 show detectable impulsive loop top emission. Considering that the finite dynamic range (about a decade) of the detection introduces a strong bias against observing comparatively weak loop top sources, we conclude that loop top emission is a common feature of all flares. We summarize the observations of the footpoint-to-loop top flux ratio and the spectral indexes. We present light curves and images of all the important newly analyzed limb flares. Whenever possible we present results for individual pulses in multipeak flares and for different loops for multiloop flares. We then discuss the statistics of the fluxes and spectral indexes of the loop top and footpoint sources, taking into account observational selection biases. The importance of these observations (and those expected from the scheduled HESSI satellite with its superior angular spectral and temporal resolution) in constraining acceleration models and parameters is discussed briefly.
\end{abstract}

Subject heading: acceleration of particles - Sun: flares — Sun: X-rays, gamma rays

On-line material: color figures

\section{INTRODUCTION}

The discovery of hard X-ray sources located at or above the top of solar flare loops by the Hard X-Ray Telescope (HXT) on board the Yohkoh satellite has provided great insight into the processes that drive solar flares. The canonical event for this phenomenon is the flare of 1992 January 13, first described by Masuda et al. (1994) and later analyzed by Alexander \& Metcalf (1997), commonly referred to as the "Masuda" flare. This flare, which is clearly delineated by a soft X-ray (thermal) loop, shows three compact hard X-ray sources, two located at the footpoints (FPs) and a third above the top of the soft X-ray loop. The first systematic study of such sources was undertaken by Masuda (1994). During the period of time between the satellite's first scientific observations (1991 October) up to 1993 September, Masuda selected $11 \mathrm{X}$-ray-bright limb flares observed by Yohkoh that met his selection criteria (see below); one of these 11 events was an interrupted event and was not analyzed further. Of the remaining 10 flares, six demonstrated a nonthermal loop top (LT) source while another demonstrated what Masuda termed a "superhot" thermal LT source. This indicates that LT hard X-ray emission is fairly common. This view is strengthened further considering the limited dynamic range of the HXT instrument and the results that we describe in this paper. Thus, it is reasonable to conclude that LT sources are present in all flares.

\footnotetext{
${ }^{1}$ Also, Departments of Physics, Applied Physics and Astronomy Program.

${ }^{2}$ Current address: Department of Physics, University of Chicago, Chicago, IL 60637.
}

It is generally agreed that the primary energy of solar flares must come from reconnection of stressed magnetic fields, and as pointed out by Masuda et al. (1994), the Yohkoh LT observations lend support to theories that place the location of energy release high up in the corona. The energy released by reconnection can be used to heat the ambient plasma and/or to accelerate electrons and protons to relativistic energies. The power-law hard X-ray spectra seen in many of the LT sources indicate that nonthermal processes, such as particle acceleration, are indeed occurring at or near these locations. The exact mechanism of the acceleration is a matter of considerable debate. In previous works (see Petrosian 1994, 1996), we have argued that among the three proposed particle acceleration mechanisms (electric fields, shocks, and plasma turbulence or waves) the stochastic acceleration of ambient plasma particles by plasma waves provides the most natural means of explaining the observed spectral and spatial features of solar flares.

In two recent works (Petrosian \& Donaghy 1999, 2000, hereafter PD) we demonstrated how the power-law spectral indexes and emission ratios (obtained by Masuda 1994) can be used to constrain the model parameters. In this paper, we expand and extend Masuda's analysis for further investigation of the ubiquity and nature of the LT source and to gain a clearer picture of the range of values of the fluxes and spectral indexes of the FP and LT sources. Furthermore, we investigate the temporal evolutions of the images of several new flares observed by HXT to determine the relationship between the many spatially and temporally distinct sources that occur in complex flaring events. In the next section we describe our procedure, and in $\S 3$ we present the light curves 
and images of most of the new events, including three events that appear to be examples of multiple-loop flares. In $\S 4$ we present the statistics of the relative fluxes and spectral indexes of the LT and FP sources. In $\S 5$ we present a summary and our conclusions.

\section{PROCEDURE}

In seeking to expand the sample of flares with loop top emission, we have searched through the Yohkoh HXT Image Catalogue (Sato et al. 1998) for appropriate limb flares using Masuda's two criteria:

1. Heliocentric longitude greater than $80^{\circ}$. This ensures maximum angular separation between LT and FP sources.

2. Peak count rate in the M2 band greater than 10 counts per second per subcollimator (counts $\mathrm{s}^{-1} \mathrm{SC}^{-1}$ ). This ensures that at least one image can be formed at high energies $(\sim 33-53 \mathrm{keV})$, where thermal contribution is expected to be lower.

In total, we found 20 events from 1991 October through 1998 August that satisfy these conditions. Of the events before 1993 September (when Masuda stopped his search), we found 12 such flares, including 11 events noted by Masuda and one event (that of 1991 December 18) that satisfies the search criteria but was apparently overlooked by Masuda. From the period after 1993 September up to 1998 August (when the Image Catalogue was finished), we have an additional eight events. In the interest of completeness we include all 20 events in this survey (see Table 1). However, two events (1992 November 2, noted but not analyzed by Masuda, and 1998 May 10) have their observations interrupted by spacecraft night, and we are unable to obtain any useful information from them. An interesting aside (which we shall return to in $\S 4$ ) is that of the nine new events, three appear to be examples of interacting loop structures with multiple LT and FP sources, of the type analyzed by Aschwanden et al. (1999). It is surprising that none of the 11 Masuda events are in this category. It should also be noted that three of the flares observed before 1993 September show no detectable LT emission, while all of the flares since that date (with the exception of one ambiguous source) show LT emission.

For the 10 early events, Masuda quotes LT and FP spectral indexes for the events when those sources are detected, although he does not supply the time intervals or spatial regions over which the indexes are taken. Masuda obtains two spectral indexes by fitting a power law to the $\mathrm{L}(\sim 13-23$ $\mathrm{keV}$ ) and M1 ( 23-33 keV) band fluxes and the M1 and M2 band fluxes. We carry out spectral fitting both to a simple power law as well as a broken power law using all four channel fluxes (including the $\mathrm{H}$ band with energy range $~ 53-93$ $\mathrm{keV}$ ), whenever significant fluxes in all four bands are detected. These results are summarized in Tables 2 and 3.

For two events (1992 January 13 and 1992 October 4), Masuda performs a temporal and spatial analysis, provid-

TABLE 1

List of Events from 1991 October to 1998 August That Satisfy Masuda's Two Criteria

\begin{tabular}{|c|c|c|c|c|c|c|}
\hline $\begin{array}{l}\text { Event Number } \\
\text { (1) }\end{array}$ & $\begin{array}{l}\text { Date } \\
(2)\end{array}$ & $\begin{array}{l}\text { Peak Time } \\
\text { (UT) } \\
\text { (3) }\end{array}$ & $\begin{array}{l}\text { Disk Position } \\
\text { (4) }\end{array}$ & $\begin{array}{c}\text { GOES Class } \\
(6)\end{array}$ & $\begin{array}{l}\text { L-Band Peak Count } \\
\text { (7) }\end{array}$ & $\begin{array}{c}\text { Loop Top Location } \\
\text { (8) }\end{array}$ \\
\hline \multicolumn{7}{|c|}{ Masuda (Pre-1993 September) Flares } \\
\hline 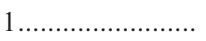 & 1991 Dec 2 & $04: 55: 27$ & N18E84 & M3.6 & 61 & Inside* \\
\hline 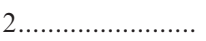 & 1991 Dec 15 & 02:44:11 & $\ldots$ & M1.2 & 30 & No SXT \\
\hline 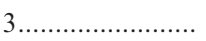 & 1991 Dec 18 & $10: 27: 40$ & S14E84 & M3.5 & 379 & Inside (new) \\
\hline $4 \ldots \ldots \ldots \ldots \ldots \ldots \ldots \ldots$ & 1992 Jan 13 & $17: 29: 37$ & S16W84 & $\mathrm{M} 2.0$ & 51 & Above* \\
\hline 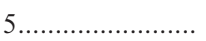 & 1992 Feb 6 & $03: 25: 11$ & N06W84 & M7.6 & 807 & Inside (superhot) \\
\hline 6 & $1992 \mathrm{Feb} 17$ & $15: 42: 09$ & N16W80 & M1.9 & 33 & Inside \\
\hline 7 & 1992 Apr 1 & 10:14:07 & 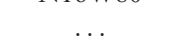 & $\mathrm{M} 2.3$ & 50 & No LT source* \\
\hline 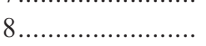 & 1992 Oct 4 & $22: 21: 07$ & S05W90 & $\mathrm{M} 2.4$ & 35 & Above \\
\hline 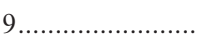 & 1992 Nov 2 & 03:00:02 & S24W84 & X9.0 & 11608 & Int. \\
\hline $10 \ldots \ldots \ldots \ldots \ldots \ldots \ldots$ & 1992 Nov 5 & $06: 19: 59$ & S18W84 & $\mathrm{M} 2.0$ & 68 & None \\
\hline $11 \ldots \ldots \ldots \ldots \ldots \ldots \ldots$ & 1992 Nov 23 & 20:26:02 & S08W84 & M4.4 & 94 & No SXT \\
\hline $12 \ldots \ldots \ldots \ldots \ldots \ldots \ldots \ldots \ldots \ldots \ldots \ldots$ & $1993 \mathrm{Feb} 17$ & $10: 36: 30$ & S07W87 & M5.8 & 229 & Above \\
\hline \multicolumn{7}{|c|}{ Our Post-1993 September Flares } \\
\hline $13 \ldots \ldots \ldots \ldots \ldots \ldots \ldots$ & 1993 Sep 27 & $12: 08: 39$ & N08E84 & M1.8 & 70 & Above \\
\hline 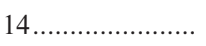 & 1993 Nov 30 & $06: 03: 37$ & S20E84 & $\mathrm{C} 9.2$ & 57 & $\mathrm{C}$ \\
\hline $15 \ldots \ldots \ldots \ldots \ldots \ldots \ldots \ldots$ & 1998 Apr 23 & $05: 44: 54$ & S20E84 & $\mathrm{X} 1.2$ & 1496 & Single* \\
\hline $16 \ldots \ldots \ldots \ldots \ldots \ldots \ldots \ldots$ & 1998 May 8 & $01: 58: 47$ & S16W84 & M3.1 & 116 & $\mathrm{C}$ \\
\hline $17 \ldots \ldots \ldots \ldots \ldots \ldots \ldots \ldots$ & 1998 May 9 & $03: 26: 41$ & S16W84 & M7.7 & 860 & No SXT \\
\hline 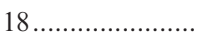 & 1998 May 10 & $13: 18: 49$ & S28E84 & M3.9 & 138 & Int. \\
\hline 19 & 1998 Aug 18 & $08: 21: 41$ & N34E84 & $\mathrm{X} 2.8$ & 3499 & Inside? \\
\hline $20 \ldots \ldots \ldots \ldots \ldots \ldots \ldots$ & 1998 Aug 18 & $22: 15: 34$ & N30E84 & $\ldots$ & 12397 & $\mathrm{C}$ \\
\hline
\end{tabular}

NotES.-Dates, UT times, position on the solar disk, flare classifications, and the peak counts in the L band (Cols. [2]-[6]) are taken from Sato et al. 1998. Col. (7) describes the location of the LT HXT source relative to the SXT loop and some other characteristics of the images. Flares identified by an asterisk $\left(^{*}\right)$ may be partially occulted by the solar limb. "No SXT" implies absence of SXT image of the loop. Flare 3, labeled "new," was missed by Masuda. Flare 4 was classified by Masuda as a superhot thermal source. Flare 15 (identified as "Single*") shows the presence of only one resolved source, which may be the LT source of an occulted loop. "C" = complex multiple loop event. "Int." = interrupted observation, not analyzed. 
TABLE 2

Spectral Parameters from Fits to Four Models of Events with Detectable H-Band Images

\begin{tabular}{|c|c|c|c|c|c|c|c|c|c|c|c|c|c|}
\hline \multirow{2}{*}{$\begin{array}{c}\text { EVEnt } \\
\text { Number }\end{array}$} & \multirow[b]{2}{*}{ Date } & \multirow{2}{*}{$\begin{array}{l}\text { Time } \\
\text { (UT) }\end{array}$} & \multirow[b]{2}{*}{ SOURCE } & \multicolumn{2}{|c|}{$\begin{array}{l}\text { Power } \\
\text { LaW } 1\end{array}$} & \multicolumn{2}{|c|}{$\begin{array}{l}\text { Power } \\
\text { LAw } 2\end{array}$} & \multicolumn{4}{|c|}{$\begin{array}{l}\text { BRoKen PoWer } \\
\text { LAW } 1\end{array}$} & \multicolumn{2}{|c|}{$\begin{array}{l}\text { BROKEN POWER } \\
\text { LAW } 2\end{array}$} \\
\hline & & & & $\gamma$ & $\chi^{2} / \nu$ & $\gamma$ & $\chi^{2} / \nu$ & $\gamma_{1}$ & $\gamma_{2}$ & $E_{\mathrm{br}}$ & $\chi^{2}$ & $\gamma_{\mathrm{L}, \mathrm{M} 1}$ & $\gamma_{\mathrm{M} 1, \mathrm{M} 2}$ \\
\hline \multirow[t]{2}{*}{$2 \ldots \ldots \ldots \ldots$} & $1991 \mathrm{Dec} 15$ & 02:44:09 & $\mathrm{A}(\mathrm{FP})$ & 6.1 & 21 & 4.5 & 5.8 & $\ldots$ & & & & 6.8 & 3.8 \\
\hline & & & $\mathrm{B}(\mathrm{FP})$ & 4.3 & 18 & 5.1 & 16 & 3.0 & 9.9 & 42 & 0.0 & 2.9 & 3.5 \\
\hline \multirow[t]{3}{*}{$4 \ldots \ldots \ldots \ldots$} & 1992 Jan 13 & $17: 28: 04$ & $\mathrm{~A}(\mathrm{FP})$ & 3.5 & 2.2 & 3.8 & 0.32 & $\ldots$ & & & & 3.1 & 3.9 \\
\hline & & & $\mathrm{B}(\mathrm{FP})$ & 3.3 & 8.6 & 3.7 & 0.02 & $\ldots$ & & & & 2.5 & 3.7 \\
\hline & & & $\mathrm{C}(\mathrm{LT})$ & 5.3 & 12.8 & 6.6 & 0.45 & $\ldots$ & & & & 4.2 & 6.8 \\
\hline 7 & 1992 Apr 1 & 10:13:05 & $\mathrm{A}(\mathrm{FP})$ & 3.8 & 1.3 & 3.9 & 0.0 & 2.2 & 3.9 & 18 & 0.0 & 3.7 & 3.9 \\
\hline \multirow[t]{3}{*}{8} & 1992 Oct 4 & $22: 19: 31$ & $\mathrm{~A}(\mathrm{FP})$ & 4.7 & 21 & 5.9 & 0.01 & $\ldots$ & & & & 3.2 & 5.9 \\
\hline & & & $\mathrm{B}(\mathrm{FP})$ & 4.5 & 14 & 4.9 & 19 & $\ldots$ & & & & 4.0 & 3.6 \\
\hline & & & $\mathrm{C}(\mathrm{LT})$ & 5.8 & 0.61 & 5.6 & 0.85 & 5.8 & 7.6 & 56 & 1.1 & 6.0 & 5.3 \\
\hline $10 \ldots \ldots \ldots \ldots$ & 1992 Nov 5 & $06: 19: 20$ & $\mathrm{~A}(\mathrm{FP})$ & 4.1 & 20 & 4.4 & 8.2 & 3.6 & 5.0 & 34 & 0.0 & 3.6 & 4.1 \\
\hline \multirow[t]{3}{*}{$19 \ldots \ldots \ldots \ldots$} & 1998 Aug 18 & $08: 19: 13$ & $\mathrm{~A}(\mathrm{LT})$ & 7.4 & 32 & 7.4 & 64 & $\ldots$ & & & & 7.4 & 7.7 \\
\hline & & & $\mathrm{B}(\mathrm{FP})$ & 6.0 & 260 & 4.3 & 0.41 & 12 & 4.3 & 18 & 0.45 & 6.6 & 4.2 \\
\hline & & & $\mathrm{C}(\mathrm{FP})$ & 5.1 & 140 & 6.2 & 0.02 & $\ldots$ & & & & 3.9 & 6.2 \\
\hline \multirow[t]{5}{*}{$20 \ldots \ldots \ldots$} & 1998 Aug 18 & $22: 14: 36$ & $\mathrm{~A}(\mathrm{FP})$ & 3.1 & 590 & 3.5 & 200 & 2.3 & 3.9 & 32 & 0.0 & 2.2 & 3.1 \\
\hline & & & $\mathrm{B}(\mathrm{FP})$ & 3.6 & 2.4 & 3.6 & 1.3 & $\ldots$ & & & & 3.7 & 3.5 \\
\hline & & & $\mathrm{C}(\mathrm{FP})$ & 3.5 & 17 & 3.4 & 1.9 & 5.6 & 3.4 & 18 & 1.9 & 3.7 & 3.4 \\
\hline & & & $\mathrm{D}(\mathrm{LT})$ & 5.0 & 66 & 4.4 & 59 & 5.3 & 3.2 & 37 & 0.0 & 5.3 & 4.9 \\
\hline & & & $\mathrm{E}(\mathrm{LT})$ & 3.8 & 32 & 4.0 & 43 & 3.2 & 3.8 & 9.7 & 63 & 3.4 & 4.4 \\
\hline
\end{tabular}

Notes.-Power Law 1 uses all four channels (degrees of freedom $\nu=2$ ); Power Law 2 uses the three highest channels $(\nu=1)$; Broken Power Law 1 uses all four channels ( $E_{\mathrm{br}}$ in units of kilo-electron volts); and Broken Power Law 2 the three lowest bands with $E_{\mathrm{br}}$ set to $\sim 28 \mathrm{keV}$, the midpoint of the M1 channel $\left(\chi^{2}=0, \nu=0\right)$. Missing spectral parameters for events in the Broken Power Law 1 column indicate that the $\chi^{2}$ minimization for the spectra did not converge.

ing light curves for emission from the LT and FP regions. He defines a box around each source, with size and center position determined from the best available image, and then obtains light curves by measuring the total flux of photons coming from within the box as a function of time. We undertake this temporal analysis for all the events in our list for which the necessary data are available. The data reduction is done with the standard Yohkoh HXT software package, which uses the maximum entropy method (MEM); in particular, we use the "hxt_multimg," "lcur_image," and "hxt_boxfsp" routines.

For several events, we have performed image reconstruction using both the standard MEM routine as well as the "pixon" technique used by Alexander \& Metcalf (1997). As described in that paper, the pixon routine achieves significantly better photometry of faint sources and better rejection of spurious sources than the standard MEM routine, albeit with a significantly longer computational time. These features are useful to our analysis since we are often attempting to resolve a faint LT source in the presence of much stronger FP sources. In general, the pixon and MEM analyses yielded similar light curves.

Major sources of systematic uncertainty in our analysis arise from the difficulty in defining exactly what constitutes an LT or an FP source. In many cases, one or more of these sources will be quite strong and well localized at one epoch during a flare, but they may disappear, change position, or combine with another source as the flare evolves. Therefore, the procedure of integrating the fluxes using a static box to obtain a light curve for the duration of the flare can lead to erroneous conclusions because of the possibility of contamination of one source by neighboring sources. Further errors arise from the fact that in many flares nonthermal emission, which tends to be more impulsive and peak earlier, is superimposed upon the more gradual thermal emission, which peaks later. Therefore, for the study of the impulsive component (as a means of studying the acceleration mechanism), we limit our analysis of the flux ratios or spectral indexes to the early stages of the flares to minimize the influence of the thermal component. We also use the M1 channel (rather than the L channel), where the thermal effects are expected to be smaller. Clearly, we would be safer to use channels M2 and $\mathrm{H}$, but these channel have weaker emissions.

\section{CASE STUDIES OF IMAGES AND LIGHT CURVES}

In this section we present light curves and a brief analysis for all of the newly analyzed events. Unless otherwise noted, the image and light curves were taken in the M1 band using the standard MEM procedure.

\subsection{Single-Loop Flares}

Of the 18 total events, 11 appear to be morphologically similar to the canonical Masuda event, that is, a single flaring loop anchored by twin FPs and exhibiting a distinct LT source. Of course, within this type we observe a good deal of variation. We present a few case studies.

1991 December 18. - This event occurred during the early phases of the Yohkoh mission, but for reasons unknown to us it was not selected or analyzed by Masuda. The hard X-ray images initially show one bright source (at around 10:25:00), which could be either an LT or an FP source, co-aligned with the bright region of the soft X-ray flaring loop. Shortly after the start of the flare, 
TABLE 3

SPeCtral Indexes

\begin{tabular}{|c|c|c|c|c|c|c|c|}
\hline \multirow[b]{2}{*}{ EVEnt Number } & \multirow[b]{2}{*}{ Date } & \multirow{2}{*}{$\begin{array}{l}\text { TIME } \\
\text { (UT) }\end{array}$} & \multirow[b]{2}{*}{ Source } & \multicolumn{2}{|c|}{ Power Law } & \multicolumn{2}{|c|}{ Broken Power LaW 2} \\
\hline & & & & $\gamma$ & $\chi^{2} / \nu$ & $\gamma_{\mathrm{L}, \mathrm{M} 1}$ & $\gamma_{\mathrm{M} 1, \mathrm{M} 2}$ \\
\hline \multirow[t]{3}{*}{ 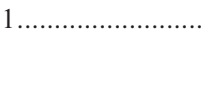 } & \multirow[t]{3}{*}{1991 Dec 2} & \multirow[t]{3}{*}{$04: 53: 45$} & $\mathrm{~A}(\mathrm{LT})$ & 6.2 & 0.6 & 6.3 & 5.9 \\
\hline & & & $\mathrm{B}(\mathrm{FP})$ & 5.8 & 3.3 & 5.2 & 7.1 \\
\hline & & & $\mathrm{C}(\mathrm{FP})$ & 5.4 & 0.01 & 5.4 & 5.3 \\
\hline \multirow[t]{3}{*}{ 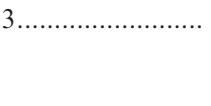 } & \multirow[t]{3}{*}{1991 Dec 18} & \multirow[t]{3}{*}{$10: 27: 16$} & $\mathrm{~A}(\mathrm{LT})$ & 7.1 & 87 & 6.5 & 9.0 \\
\hline & & & $\mathrm{B}(\mathrm{FP})$ & 6.2 & 46 & 5.8 & 7.1 \\
\hline & & & $\mathrm{C}(\mathrm{FP})$ & 8.3 & 31 & 7.9 & 11 \\
\hline \multirow[t]{3}{*}{$5 \ldots \ldots \ldots \ldots \ldots \ldots \ldots \ldots$} & \multirow[t]{3}{*}{1992 Feb 6} & \multirow[t]{3}{*}{$03: 22: 16$} & A (LT) & 8.1 & 320 & 6.8 & 12 \\
\hline & & & $\mathrm{B}(\mathrm{FP})$ & 7.7 & 43 & 6.9 & 10 \\
\hline & & & $\mathrm{C}(\mathrm{FP})$ & 8.3 & 56 & 7.6 & 11 \\
\hline \multirow[t]{3}{*}{ 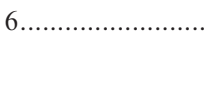 } & \multirow[t]{3}{*}{1992 Feb 17} & \multirow[t]{3}{*}{$15: 40: 44$} & A (FP) & 3.3 & 14 & 2.4 & 4.1 \\
\hline & & & $\mathrm{B}(\mathrm{LT})$ & 6.2 & 31 & 6.9 & 3.1 \\
\hline & & & $\mathrm{C}(\mathrm{FP})$ & 3.5 & 41 & 3.6 & 3.5 \\
\hline \multirow[t]{3}{*}{$11 \ldots \ldots \ldots \ldots \ldots \ldots \ldots}$. & \multirow[t]{3}{*}{1992 Nov 23} & \multirow[t]{3}{*}{$20: 24: 39$} & $\mathrm{~A}(\mathrm{FP})$ & 3.7 & 1.7 & 3.5 & 3.9 \\
\hline & & & $\mathrm{B}(\mathrm{LT})$ & 10 & 2.0 & 10 & 8.0 \\
\hline & & & $\mathrm{C}(\mathrm{FP})$ & 6.4 & 3.2 & 6.7 & 5.5 \\
\hline \multirow[t]{3}{*}{$12 .}$. & \multirow[t]{3}{*}{1993 Feb 17} & \multirow[t]{3}{*}{$10: 36: 13$} & $\mathrm{~A}(\mathrm{FP})$ & 4.8 & 37 & 4.3 & 5.6 \\
\hline & & & $\mathrm{B}(\mathrm{FP})$ & 6.5 & 42.0 & 6.0 & 7.9 \\
\hline & & & $\mathrm{C}(\mathrm{LT})$ & 6.2 & 12 & 5.8 & 7.0 \\
\hline \multirow[t]{3}{*}{$13 \ldots \ldots \ldots \ldots \ldots \ldots \ldots$} & \multirow[t]{3}{*}{1993 Sep 27} & \multirow[t]{3}{*}{$12: 08: 10$} & $\mathrm{~A}(\mathrm{LT})$ & 6.2 & 8.3 & 5.6 & 9.7 \\
\hline & & & $\mathrm{B}(\mathrm{FP})$ & 4.9 & 20 & 4.1 & 7.2 \\
\hline & & & $\mathrm{C}(\mathrm{FP})$ & 5.7 & 23 & 5.7 & 7.2 \\
\hline \multirow[t]{4}{*}{$14 \ldots \ldots \ldots \ldots \ldots \ldots$} & 1993 Nov 30 & 06:03:33 & $\mathrm{A}(\mathrm{FP})$ & 2.7 & 37 & 1.6 & 3.5 \\
\hline & & & $\mathrm{B}(\mathrm{FP})$ & 3.8 & 3.6 & 3.2 & 4.4 \\
\hline & & & $\mathrm{C}(\mathrm{FP})$ & 3.5 & 74 & 1.3 & 5.7 \\
\hline & & & $\mathrm{D}(\mathrm{LT})$ & 3.6 & 18 & 2.1 & 5.2 \\
\hline $15 \ldots \ldots \ldots \ldots \ldots \ldots$ & 1998 Apr 23 & $05: 38: 46$ & $\mathrm{~A}(\mathrm{LT})$ & 7.5 & 5.2 & 7.4 & 7.8 \\
\hline 16 & 1998 May 8 & $01: 58: 29$ & $\mathrm{~A}(\mathrm{FP})$ & 4.7 & 35 & 4.7 & 4.5 \\
\hline & & & $\mathrm{C}(\mathrm{LT})$ & 5.8 & 22 & 5.0 & 7.5 \\
\hline & & $01: 57: 28$ & $\mathrm{~B}(\mathrm{FP})$ & 3.9 & 4.0 & 3.7 & 4.2 \\
\hline & & & $\mathrm{D}(\mathrm{FP})$ & 4.2 & 19 & 3.3 & 5.5 \\
\hline & & & $\mathrm{E}(\mathrm{LT})$ & 5.5 & 22 & 4.8 & 7.0 \\
\hline $17 \ldots \ldots \ldots \ldots$ & 1998 May 9 & 03:19:36 & $\mathrm{A}(\mathrm{LT})$ & 6.6 & 5.2 & 6.1 & 7.9 \\
\hline & & & $\mathrm{B}(\mathrm{FP})$ & 6.3 & 20 & 5.9 & 7.3 \\
\hline
\end{tabular}

Notes.-Spectral indexes of events with undetected H-band images from a simple power law fit to three available bands $(\nu=1)$ and from a broken power law fit, where we fix the break energy to correspond to the midpoint of the $\mathrm{M} 1$ band at $\sim 28 \mathrm{keV}\left(\chi^{2}=0, \nu=0\right)$.

the single source splits into two and sometimes three unresolved sources co-aligned with the flaring loop. The loop joining sources $\mathrm{A}, \mathrm{B}$, and $\mathrm{C}$ in the right panel of Figure 1 is most likely an asymmetric loop. This can give rise to the observed differences between the intensities and heights above the solar limb of the two FP sources B and $\mathrm{C}$. The left panel of Figure 1 shows the light curves for each of these three regions. The dominance of source A during the initial peak of the flare could be due to efficient trapping of the accelerated electrons at the acceleration site (assumed to be at the top of the loop) by a high density of plasma turbulence. Therefore, we assume it to be an LT source for this analysis. Note that for this flare the ratio of the sum of the counts of the two FPs to that of the LT source, $\mathscr{R}=\mathrm{FPs} / \mathrm{LT}$, is about 1 , changing from 0.6 at the beginning to $\sim 1.2$ during the main pulse. In our statistical analyses described in the next section, we will often use the counts FPs and LT of the main pulse, and in few cases we use the counts of well-defined pulses separately. We will rarely use the counts or the ratios obtained during the decaying phase, where thermal contamination may become large. The pixon reconstruction of the same event shows cleaner images but the same light curves.

1993 September 27.-The Soft X-Ray Telescope (SXT) images for this event show a compact flaring loop on the limb, and the HXT images primarily show two bright FPs associated with this loop. The LT source is weaker (the count ratio $\mathscr{R} \sim 8$ ) and appears only intermittently high above the flaring loop in the corona. However, as shown in Figure 2 the LT and FP sources have similar pulse structures.

1998 August 18, 08:21 UT.-This very bright X-class flare is the first of two bright flares from this active region on this day. The SXT images show a bright loop structure that is co-aligned with two FP HXT sources and one bright LT HXT source (Fig. 3). These three sources persist throughout the impulsive phase of the flare and share common impulsive peaks. In the late phase (after 08:20:15), the LT comes to dominate and the 

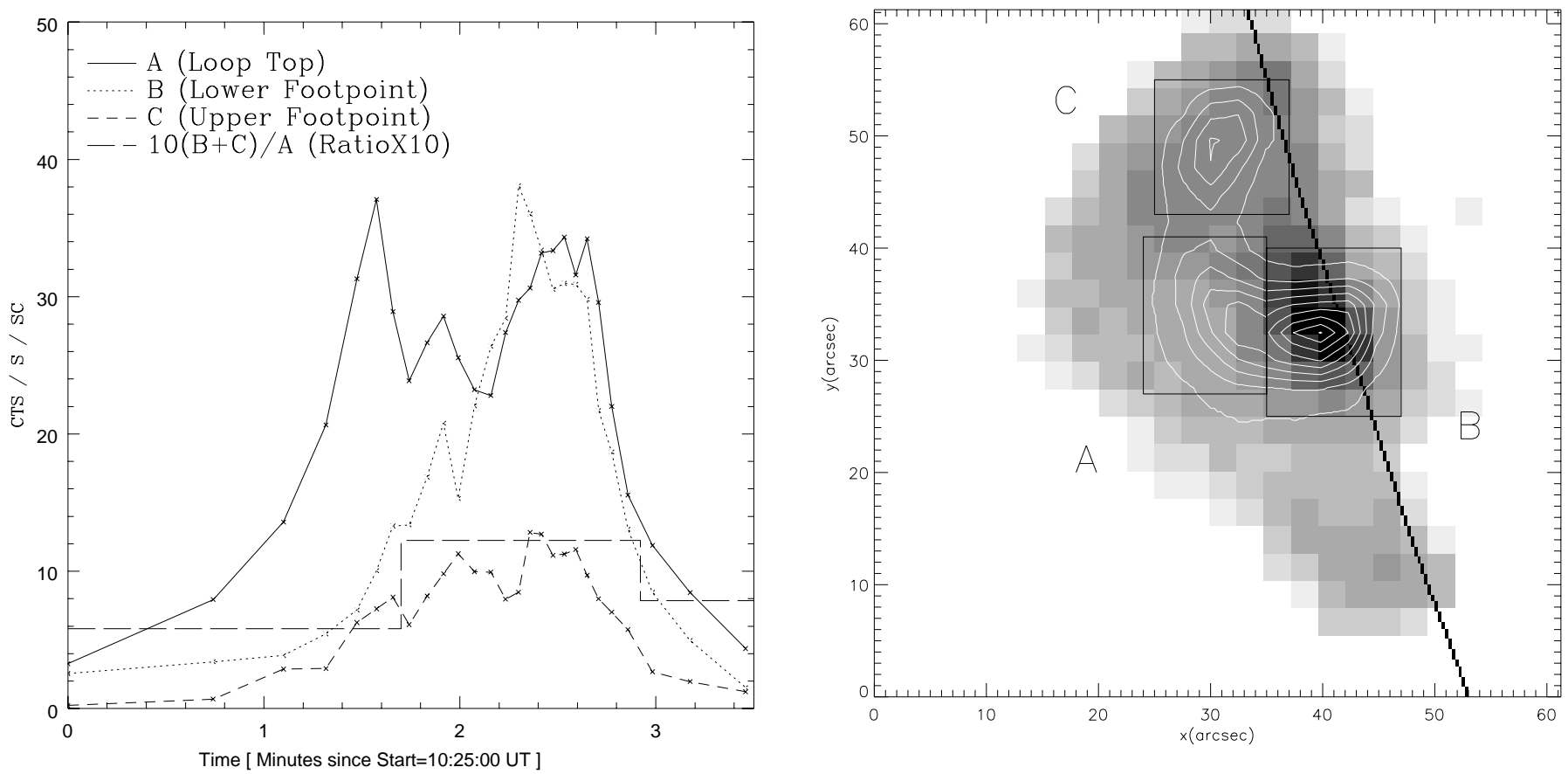

FIG. 1.-Images (right panel; HXT image times 10:27:06.9-10:27:10.04 UT) and light curves (left panel) for the 1991 December 18 flare. The contours and the gray scale show the HXT (channel M2; 33-53 keV) and SXT images of the loop, respectively, for the specified time. The diagonal line shows the location of the solar limb. The brightest contour in the HXT image is at $B_{\max }=8.1$ counts pixel ${ }^{-1}$ with $2.5 \mathrm{arcsec}^{2}$ size pixels, and the contour separations are $\Delta B=0.73$ counts pixel ${ }^{-1}$. The light curves of the LT and FP sources refer to the counts integrated over regions shown on right panel. The dashed histogram shows the average of the ratio of counts of two FPs to the LT sources, $\mathscr{R}=\mathrm{FPs} / \mathrm{LT}$ (multiplied by 10) for three time intervals. [See the electronic edition of the Journal for a color version of the left panel.]

HXT emission becomes more localized. In contrast to the preceding flare, the LT source here is extremely bright and long lived.

Taken together with the Masuda flare of 1992 January 13 and the other single-loop flares analyzed by Masuda, we note that the LT can manifest itself in many different forms. Some flares show LT emission only very briefly or very faintly. In larger flares, we observe LT sources that are clearly spatially separated from the FP sources yet have similar impulsive time structure.
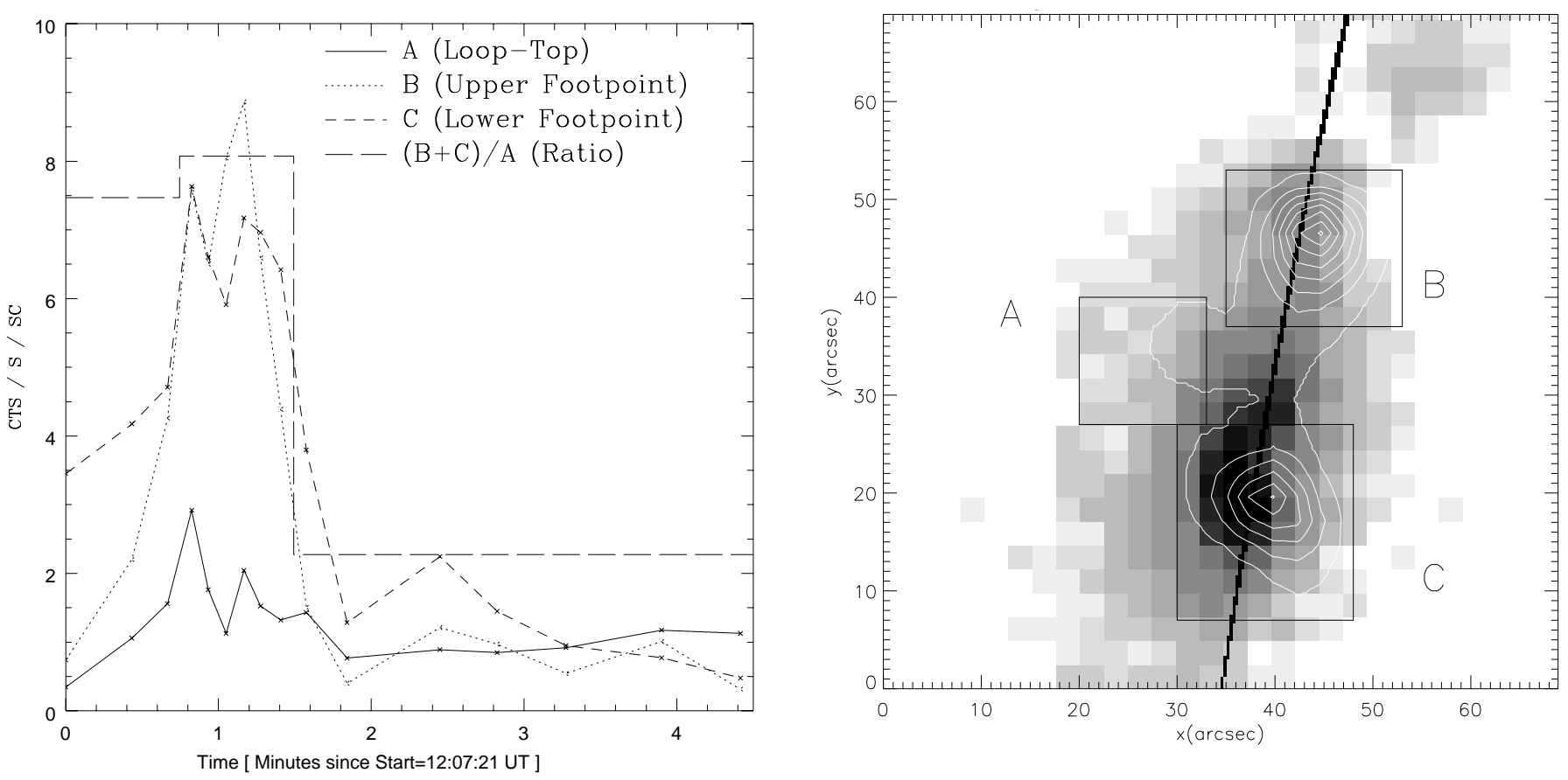

Fig. 2.- Same as Figure 1 for the 1993 September 27 flare (HXT image times 12:08:10.7-12:08:17.2 UT), with $B_{\max }=4.1$ and $\Delta B=0.41$ counts pixel ${ }^{-1}$. [See the electronic edition of the Journal for a color version of the left panel.] 

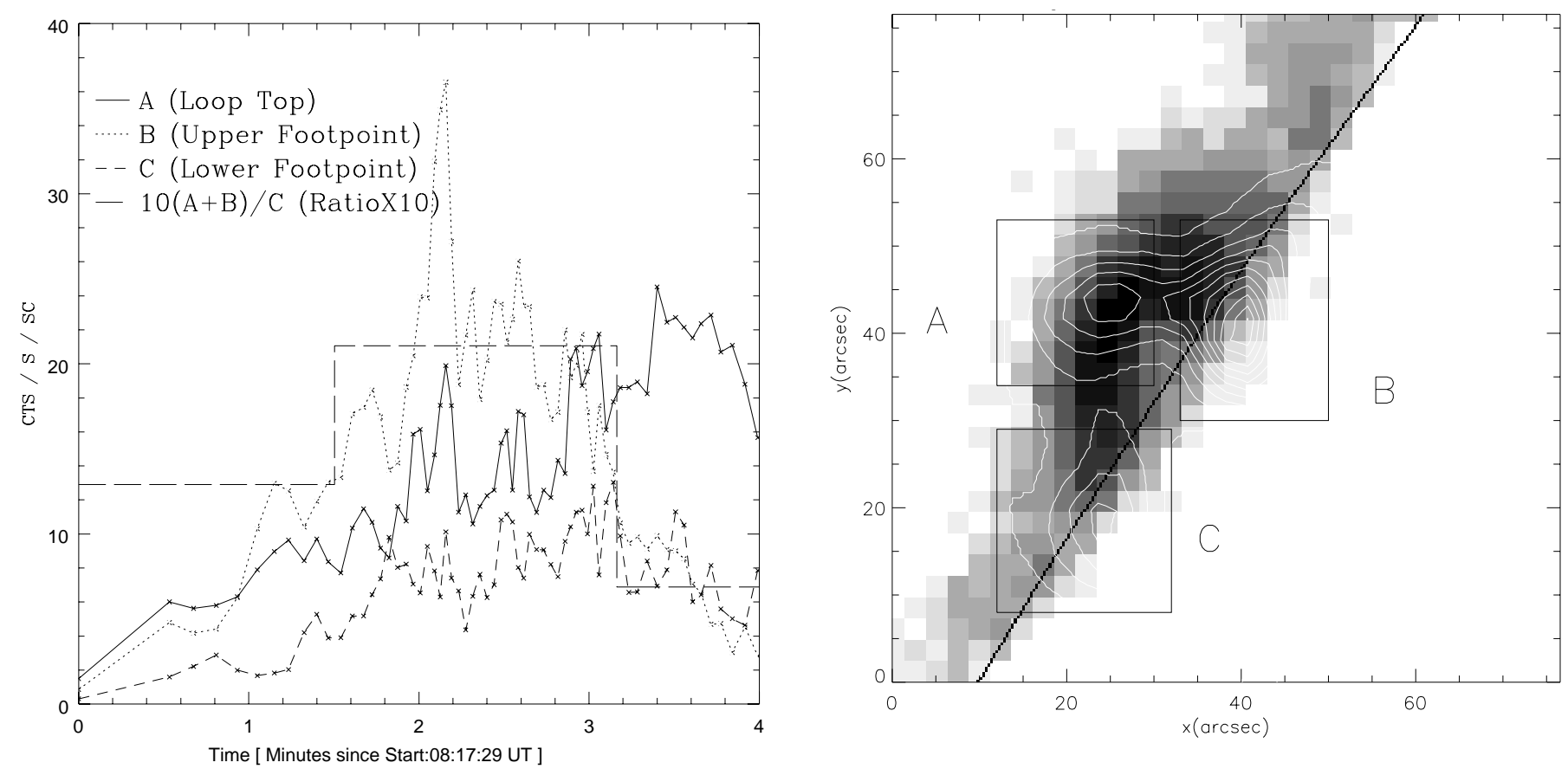

FIG. 3.-Same as Fig. 1 for the first 1998 August 18 flare (HXT image times 08:19:50.9-08:19:53.4 UT), with $B_{\max }=1.9$ and $\Delta B=0.15$ counts pixel $^{-1}$. [See the electronic edition of the Journal for a color version of the left panel.]

\subsection{Multiple-Loop Flares}

Three of the post-1993 flares that we have analyzed show HXT (and SXT) images with complex morphologies that have strong fluxes coming from several distinct regions that brighten and dim at different times. These flares cannot be modeled by a single loop and provide evidence for the existence of multiple-loop structures. It should be noted that none of the pre-1993 flares showed such complex structures.

1998 August 18, 22:15 UT.-This event (see Fig. 4) is the second flare occurring on this day in this active region, and it is one of the most energetic flaring events observed since Yohkoh's launch. This X-class flare appears in the hard Xrays as two flaring loops sharing a common central FP but is too bright for most of the flare duration to yield useful images in soft X-rays. The early SXT images have many overexposed pixels and the later images show only one flaring loop associated with the upper two FPs. The upper loop is associated with two bright FPs (B and C) and an LT scource (D), which shows impulsive behavior superimposed on an extremely hot thermal component (seen even in the M2 band). A third FP source (A) lies further south from the soft X-ray flaring loop and is associated with a comparatively fainter LT source (E) situated between it and the middle FP. The light curves give evidence for multiple acceleration sites. For example, while all three FPs participate in the first large peak (just after 22:15:05), only the upper sources show strong emission at the later peaks (22:15:30 and 22:15:50), while the lower sources fade away.

1998 May 8.-Figure 5 shows the hard X-ray image (at 01:57:40) and the corresponding light curves for this flare. The light curves and images presented here are from the pixon reconstruction method, which can identify faint sources better than the MEM method. Evidence from the soft Xray images points to the existence of two flaring loops that share the lower FP (denoted by B in the figure) and whose LT sources (E and C) overlap. The smaller loop is brighter and is associated with the FPs B and D and the LT source E. The fainter, outer loop is associated with B and the considerably fainter FP source, A, as well as the LT source C. While two upper FP sources (D and A) are indeed fainter, they do demonstrate the same impulsive variation as their brighter LT and FP relatives. Looking at the light curves, we can see that the inner loop is strongly associated with the first impulsive peak at 01:57:30. The outer loop peaks later (at 01:58:30), at a time that coincides with a low point in the light curves of the inner loop sources. It is difficult to demonstrate the presence of a causal connection, if any, between these loops. The time profile of source B is approximately a superposition of the time profile of the two FPs A and C that are separated spatially and temporally.

1993 November 30.-This event appears as a double loop structure in soft X-rays with apparent multiple LT and FP sources appearing in the hard X-ray images.

This flare was analyzed by Aschwanden et al. (1999) in their study of quadrupolar magnetic reconnection events. Aschwanden et al.'s analysis shows the presence of only a double loop with the lower FP common to both the larger outer loop and the smaller inner loop. Our analysis of this flare using the pixon method resulted in images that were considerably cleaner than those from the MEM technique. In Figure 6 we present a map of the X-ray images as well as light curves for the selected sources obtained from the pixon method. Based on these one may deduce that there are three separate loops BGC, AFE, and ADC with common FPs A and $\mathrm{C}$ having composite time profiles.

However, the basic structure of the flare remains complicated. In the early stages of the flare there are actually four FP HXT sources that correspond to four bright spots in the SXT images (sources A, B, C, and E in the figure). More 

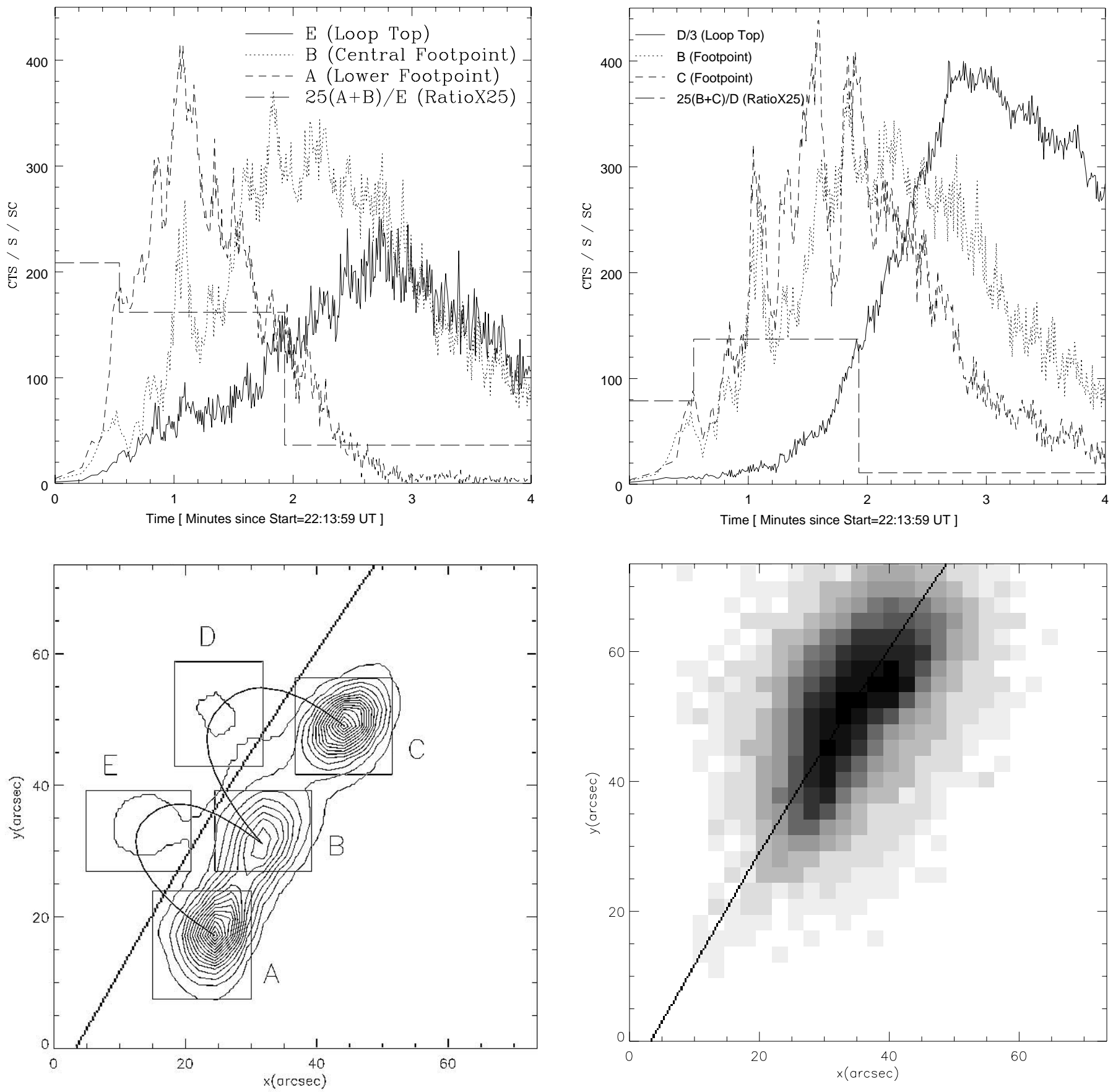

FIG. 4.-Same as Fig. 1 for the second 1998 August 18 flare. The upper left and right panel light curves represent the southern (AEB) and the northern (BDE) loops, respectively. Note that for the LT source D we plot counts divided by 3. In the HXT image (lower left panel; HXT image times 22:15:05.822:15:06.3 UT) $B_{\max }=14.8, \Delta B=0.82$ counts pixel $^{-1}$, the diagonal line shows the limb location, and the two arcs sketch the presumed loop outlines. The SXT image is shown separately in the lower right panel (SXT image time 22:16:51.8 UT) to avoid confusion. [See the electronic edition of the Journal for a color version of the upper panels.]

confusing is the behavior of what appear to be three separate sources appearing high in the corona (sources $\mathrm{D}, \mathrm{F}$, and G). The fainter source (D) appears early in the flare at the very top of the loop, while the two stronger sources (F and G) appear later and at lower altitudes. As the flare evolves, all of these FP and LT sources shift their position with respect to each other, making it very difficult to perform the usual analysis. A comparison of the light curves for sources A, C, and D (the outer loop) shows that they peak at similar times early in the impulsive phase. However, the peaks of the light curves for other sources are not correlated as clearly and have extremely complicated structures. Note also that the LT sources in this flare are much weaker than the FP sources.

\subsection{Miscellany}

1998 April 23.-This flare appears in the soft X-ray images as bright diffuse emission above the solar limb, accompanied by thin, quickly evolving loop structures that are probably not the main flaring loop. The HXT emission initially appears in two sources but quickly become one 

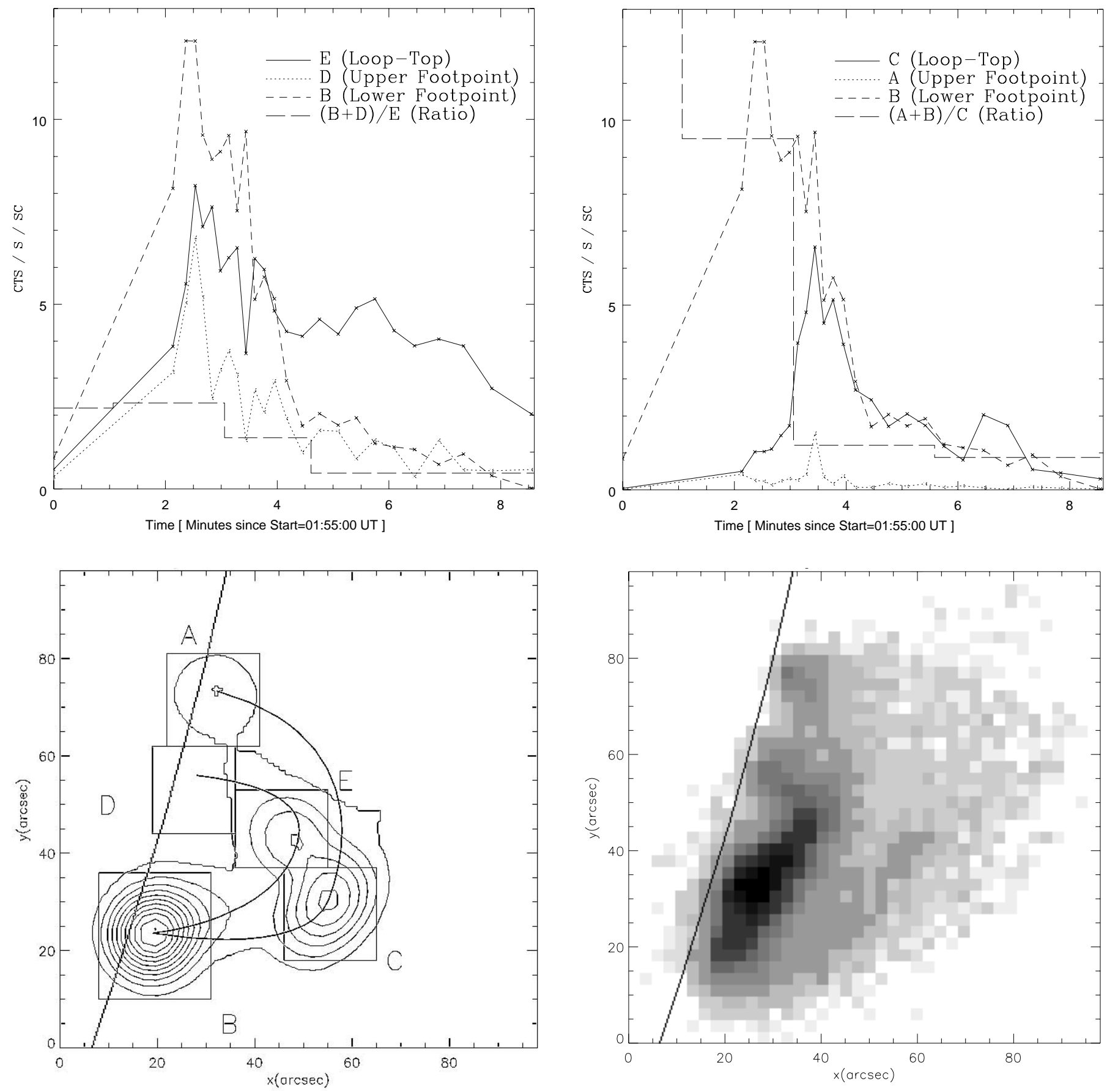

FIG. 5.-Same as Fig. 4 for the 1998 May 8 flare. The upper left and right panel light curves represent the inner (DEB) and the outer (ACB) loops, respectively. In the HXT image, $B_{\max }=3.0$ and $\Delta B=0.23$ counts pixel $^{-1}$ (HXT image times 01:58:26.7-01:58:36.2 UT; SXT image time 01:58:05.7 UT). These images were reconstructed using the pixon method. [See the electronic edition of the Journal for a color version of the upper panels.]

undifferentiated source associated with the bright diffuse soft X-ray emission. The lack of an observable flaring loop indicates the possibility that this flare occurred behind the limb and the emission we are seeing is very high up in the corona, rather than a single FP source (see Fig. 7).

1998 May 9.- This flare appears as a single FP and an LT hard X-ray source only during the initial 2 minutes of the flare (see Fig. 8), when we see one strong FP accompanied by much fainter emission above it that may be an LT source. As the flare progresses, the FP source grows in intensity so much that the limited dynamic range of the HXT makes the detection of the much fainter LT signal impossible. The dis- crepancy between the positions of the SXT and HXT images is large, so that it is difficult to determine whether the observed HXT sources are associated with any SXT features.

\section{STATISTICAL RESULTS}

In this section we consider the statistical properties of the flares listed in Table 1 and summarize the differences between the original Masuda flares and the new events classified by us. In total, there are 18 events for which we have sufficient data. Of these, 15 show at least one impulsive 

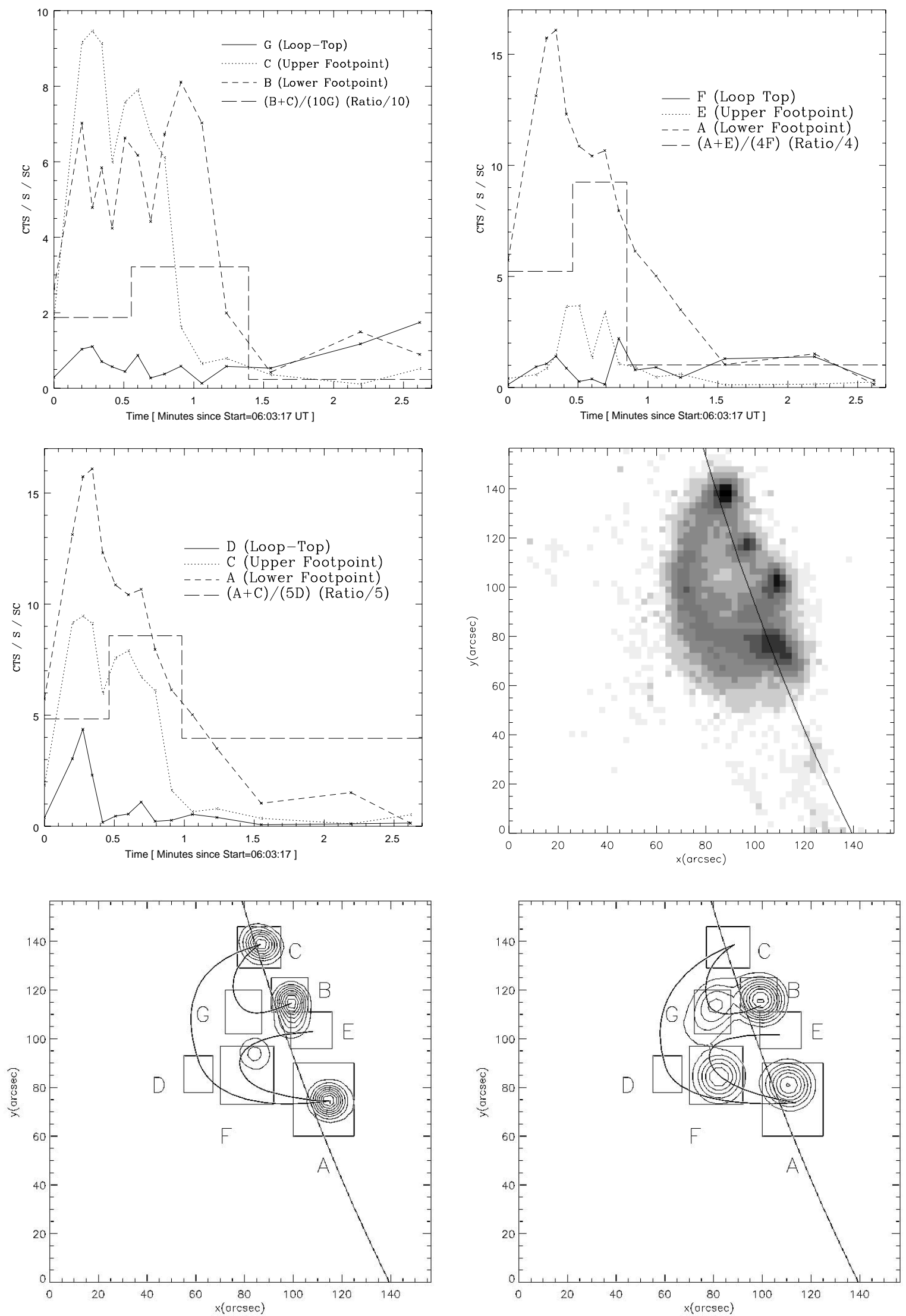

FIG. 6. - Same as Fig. 4 for the 1993 November 30 flare. The light curves in the middle left panel represents what may be the outer flaring loop (ADC), while those in the upper two panels represent other sources seen in the flare, which may form two inner loops (CGB and EFA). The SXT image is shown in middle right separately (SXT image time 06:03:57 UT), and two HXT images at two different times are shown in the lower panels, with $B_{\max }=2.5$ and $\Delta B=0.23$ counts pixel ${ }^{-1}$ for the left panel (HXT times 06:04:04-06:04:11 UT) and $B_{\max }=1.7$ and $\Delta B=0.16$ counts pixel ${ }^{-1}$ for the right panel (HXT times 06:05:2806:05:54 UT). These images were reconstructed using the pixon method. [See the electronic edition of the Journal for a color version of the upper panels and the middle left panel.] 

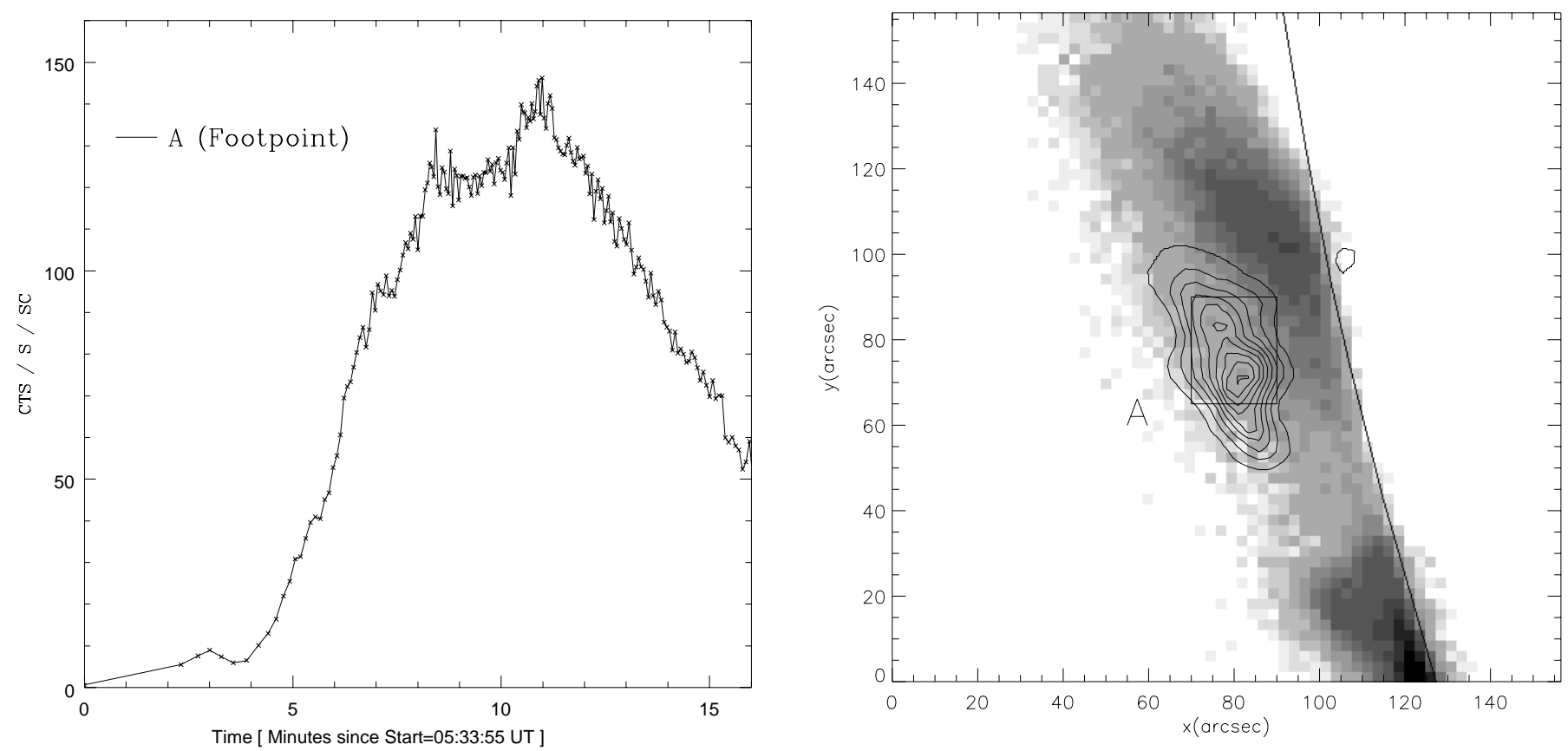

FIG. 7. - Same as Fig. 1 for the 1998 April 23 flare (HXT image time 05:38:05-05:38:19 UT), with $B_{\max }=4.4$ and $\Delta B=0.40$ counts pixel $^{-1}$. The FP sources of this flare are most likely occulted, and source A represents the LT source. [See the electronic edition of the Journal for a color version of the left panel.]

source that can be classified as an LT source. Most of these can be classified as nonthermal, and one (the 1992 February 6 flare) is classified by Masuda as a superhot thermal LT source. The remaining three show no detectable LT emission. As we shall see below, these nondetections are due to the shortcomings of the instrument, indicating that LT emission is a general feature of solar flares. We discuss the statistics of two physical characteristics, namely, the relative fluxes and spectral indexes of the LT and FP sources.

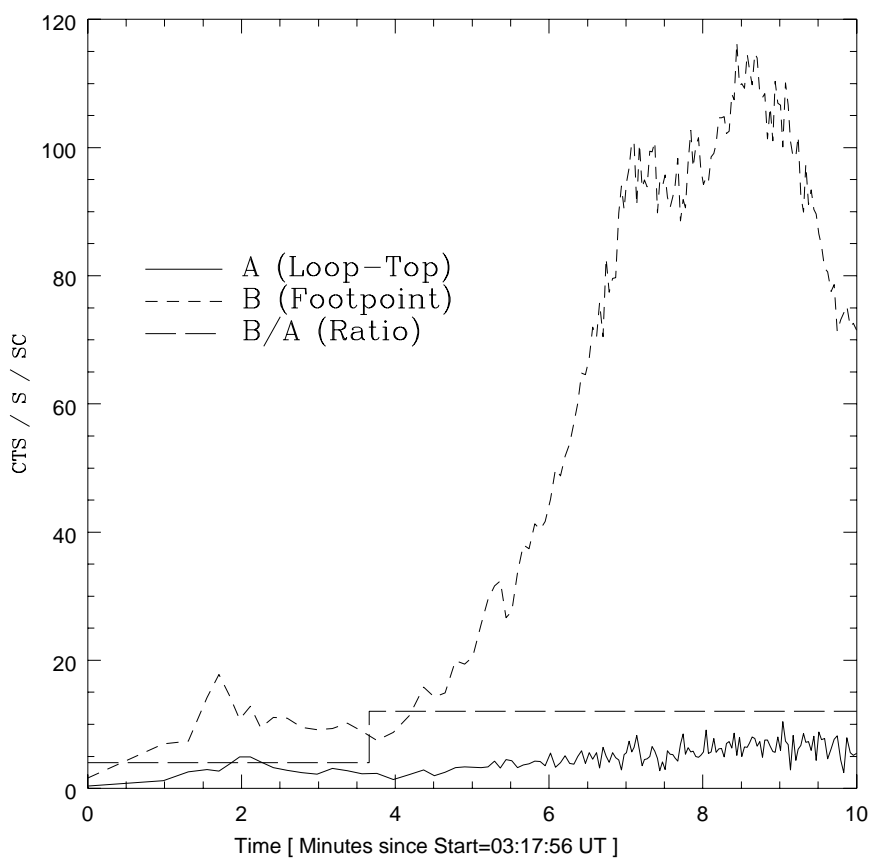

\subsection{Relative Fluxes}

The most directly available data are the statistics of the relative values and distributions of the fluxes from the LT and FP sources. Theoretically, the relative values of these emissions are important for determination of the characteristics of the models. As shown in PD, the ratio of LT to FP emission can be related to the ratio of acceleration and diffusion rates of the electrons as well as the plasma density and

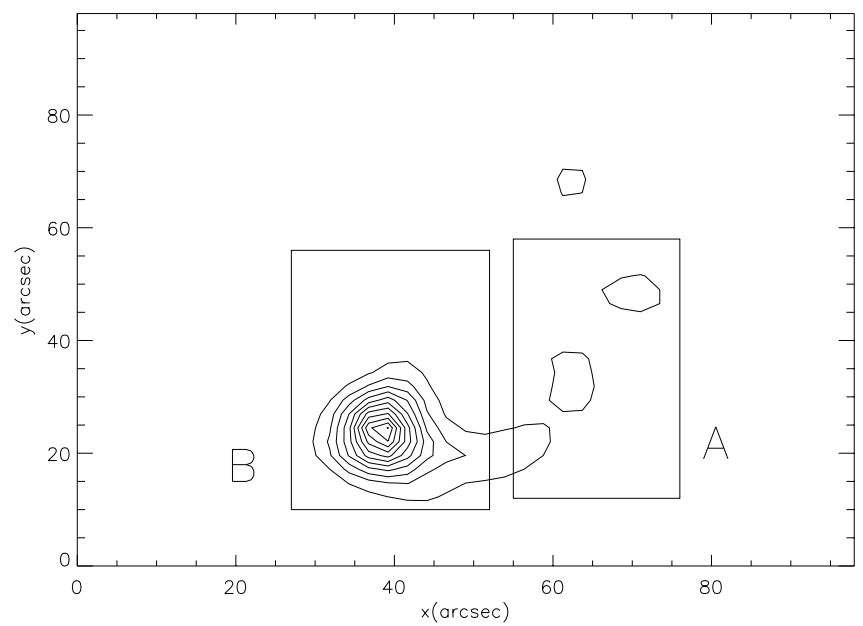

FIG. 8. - Same as Fig. 1 for the 1998 May 9 flare (HXT image times 03:19:54-03:20:03 UT), with $B_{\max }=4.1$ and $\Delta B=0.31$ counts pixel $^{-1}$. [See the electronic edition of the Journal for a color version of the left panel.] 


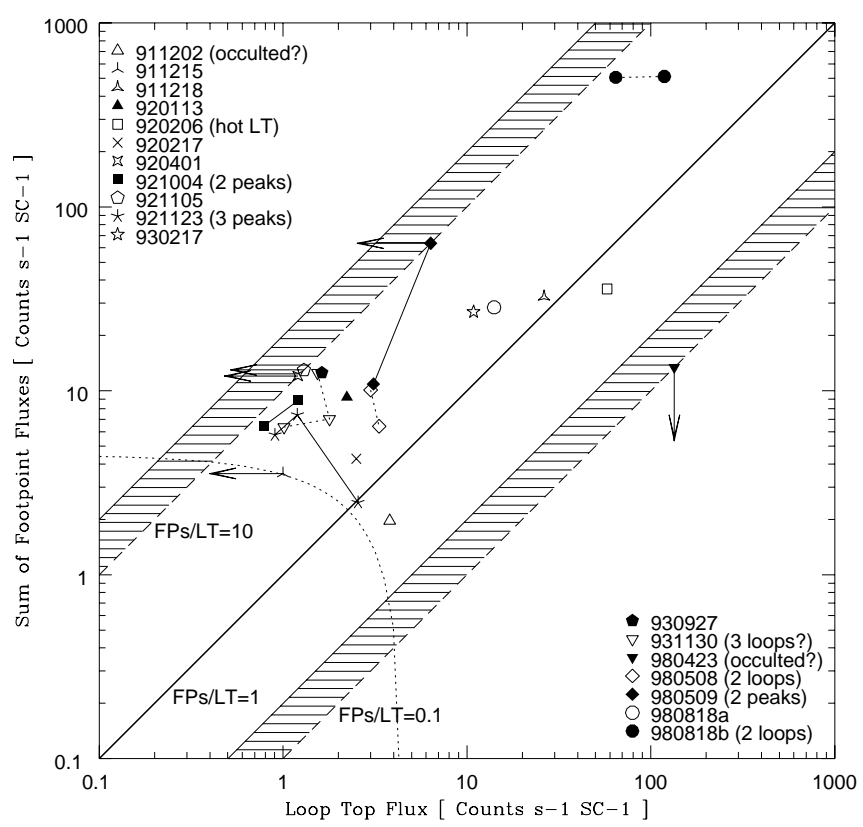

FIG. 9.- Counts from two FPs vs. LT counts, in the M1 band for all 18 flares in our sample. The diagonals represent lines of constant ratio $(\mathscr{R}=\mathrm{FPs} / \mathrm{LT})$ and represent detection thresholds arising from the finite dynamic range of the instrument: solid lines at 20 and 0.2 for two equal FPs and dotted diagonals for one dominant FP source. Flares with an undetected LT source are denoted by an arrow placed on the upper bound of detection of $\mathscr{R}=10$. The dotted curve shows the event selection threshold of 10 counts s${ }^{-1} \mathrm{SC}^{-1}$ at the M2 band, which on the average (see Fig. 10) means FPs $+\mathrm{LT} \simeq 5$ counts $\mathrm{s}^{-1} \mathrm{SC}^{-1}$ for the $\mathrm{M} 1$ band. For uniformity, all the data points on this plot were obtained using the standard MEM reconstruction procedure. [See the electronic edition of the Journal for a color version of this figure.]

magnetic field strength. Here, we examine the relative values of the LT and FP emissions for the entire sample.

In Figure 9, we plot the sum of the count rates (in units of counts $\mathrm{s}^{-1} \mathrm{SC}^{-1}$ ) of the two footpoint sources (FPS) versus the count rates of the corresponding loop top source $(L T)$. The values shown in this figure refer to count rates (or fluxes) spatially integrated over each source and integrated over the entire impulsive duration of each flare or over individual pulses in flares where distinct pulses and loops are discernible. For three events, where multiple-loop structures with corresponding LT and FP sources are observed (see $\S 3$ ), we plot LT and FP fluxes for each loop as separate points but connect them with lines.

The primary trend seen in this figure is an obvious correlation between FP and LT fluxes over more than 2 decades of flux. The second striking feature is that for most flares (or pulses in flares) the ratio of these two fluxes is confined to $1<\mathscr{R}=$ FPs $/ \mathrm{LT}<10$. Only three events lie beneath the line of equality (thick solid line). The presence of an upper envelope is most likely an artifact of the limited dynamic range of the instrument and the image reconstruction process (estimated to be about 1 decade) and is not an intrinsic feature of the flare emission. This limitation means that for two FPs with equal fluxes the plotted ratios will lie in the range $20<\mathscr{R}<1 / 5$ shown by the two thin solid diagonal lines. But if one FP is much stronger than the other, then the above ratio should lie in the range $10<\mathscr{R}<1 / 10$, shown by the dotted lines. (Because of the limited dynamic range, the weaker FP source cannot be more than 10 times weaker than the stronger one. Hence, strictly speaking the latter limits should be 11 and 0.11 .) Since the LT source that is 10 or 20 times weaker than the accompanying FPs would not be detected by the HXT instrument or revealed by the image reconstruction process, we show the three events with no detectable LT emission with horizontal arrows starting at the $\mathscr{R}=10$ line, which assumes equal counts for the two FPs. Similarly, events falling beneath the $\mathscr{R}=0.1$ (or 0.2 ) line will not have a detectable FP emission. There is only one flare with the ratio approaching this value. This is the 1998 April 24 flare, shown by a vertical arrow in Figure 9. This and one of the other two events below the $\mathscr{R}=1$ line, the 1991 December 2 flare, are believed to have occurred behind the limb, so that the FP sources are fully or partially occulted, giving it an abnormally low flux ratio. (Masuda mentions that two other events, 1992 January 13 and 1992 April 1, may also have occurred behind the limb.) The other flare below the $\mathscr{R}=1$ is the 1992 February 6 event, which according to Masuda shows a (superhot) thermal spectrum for the LT and FP sources. Our spectral analysis shows a very steep spectrum for these sources (see Table 3), supporting this assertion. The 1991 December 18 event, missed by Masuda, also shows a very steep spectrum for both LT and FP sources (see Table 3) and lies near the equality line, $\mathscr{R}=1$.

We therefore conclude that the absence of events below the line of equality $(\mathscr{R}=1)$ is not an instrumental effect and must be intrinsic to the flare process. It should be reemphasized that the limitations introduced by the dynamic range will also bias the data in favor of events with FP flux ratios of less than 10. Those with greater ratios will appear as a double (FP and LT) or just a single (FP) source depending on the strength of the LT source. We also note that for some, but not all, events with multiple pulses and/or multiple loops, the data points from different loops or pulses lie near each other (e.g., 1992 October 4 and 1998 May 8). Interesting exceptions are the flare on 1992 November 23, which exhibits one pulse with a significantly lower value of $\mathscr{R}$ than the other two pulses, and the flare on 1998 May 9, where an LT source is detected during the first pulse but not during the later, much stronger pulse (which we show in Fig. 9 with an upper bound for the LT source).

We must also consider the truncation introduced by our selection criteria. Because of the threshold of 10 counts $\mathrm{s}^{-1}$ $\mathrm{SC}^{-1}$ imposed by us (and Masuda), only flares with the flux (in the M2 band) of FPs $+\mathrm{LT}>10$ counts s${ }^{-1} \mathrm{SC}^{-1}$ will be in the sample. We estimate below that this translates to a threshold of 5 counts s${ }^{-1} \mathrm{SC}^{-1}$ for the M1 band. This truncation boundary is shown by the dotted curve in Figure 9 . As is evident, all flares except the 1991 December 15 flare fall above this boundary.

In addition to the bounds derived from our selection criterion and the finite dynamic range, there is also an absolute bound below which the instrument sensitivity drops rapidly. Since the HXT detection of flux is not based in a welldefined trigger process, we attempt to determine this bound by studying the distribution of flare peak count rates using the entire HXT database of 1307 flares over the same time period. In Figure 10, we plot the differential distribution of flare peak count rates for each of the four HXT channels and for the peak count rate summed over the four channels. From each count rate we subtract a representative background count rate for the channel. Sato et al. (1998) give approximate background count rates of $\mathrm{L} \simeq 2$, 


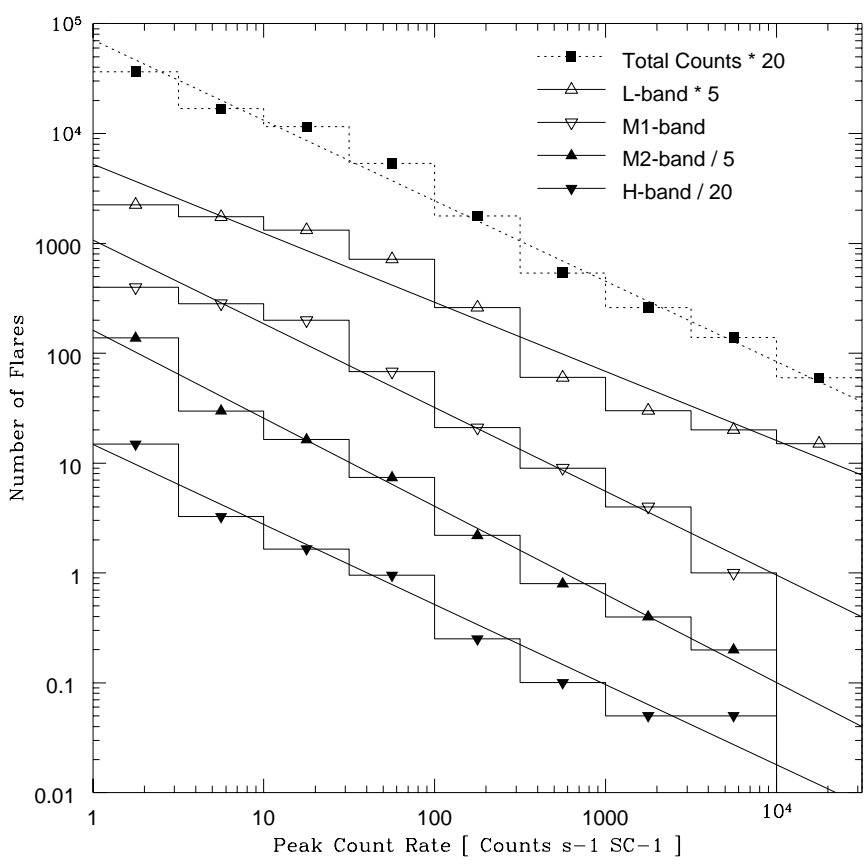

FIG. 10.-Differential distribution of peak counts of all the flares for the entire mission (1991 October-1998 August), for the sum and each of the four channels (bands L, M1, M2, and H, from top to bottom) of the HXT instrument. For clarity the histograms are shifted vertically by the indicated factors. The straight lines show the linear regression fit for each histogram. The values of the slopes $\gamma$ are $-0.73,-0.63,-0.76,-0.80$, and -0.73 from top to bottom, respectively. [See the electronic edition of the Journal for a color version of this figure.]

$\mathrm{M} 1=\mathrm{M} 2 \simeq 1$, and $\mathrm{H} \simeq 9$ counts $\mathrm{s}^{-1} \mathrm{SC}^{-1}$. As is evident, the distributions roughly follow a power law, especially at high and midrange values, in agreement with many previous flare surveys (see Lee, Petrosian, \& McTiernan 1995 and references therein). We also plot the power-law distributions from linear regression fits to the five distributions and give the power-law indexes (i.e., logarithmic slopes). The slope values of $\sim-0.6$ to -0.8 obtained here are consistent with previous results (Lee et al. 1995) and show that Yohkoh flares do not suffer from additional biases and that our results regarding the LT emission are applicable generally to all flares. The flattening away from the power law at low count rates is due to a decrease in the sensitivity of the detectors. This is especially prominent in the $\mathrm{L}$ channel, because of its higher background rates. For the M1 and M2 bands there seems to be very little flattening down to the quoted background value of 1 count s $\mathrm{s}^{-1} \mathrm{SC}^{-1}$. Note that the M2 and $\mathrm{M} 1$ band distributions are shifted relative to each other horizontally (and vertically too) by an approximately constant value of about 2 . This means that the average threshold value of the $\mathrm{M} 1$ counts $\mathrm{s}^{-1} \mathrm{SC}^{-1}$ for our sample is about 5. This value was used in determining the truncation boundary in Figure 9 (dotted curve).

In summary, from the above analysis of the relative strengths of the LT and FP fluxes we can conclude that the hypothesis that all flares have LT emission is consistent with the extant, though limited, data. Figure 11 shows the distribution of $\mathscr{R}=$ FPs/LT for the flares presented in Figure 9. This distribution is fairly flat between 0.5 and 8 , and there are four flares with undetected LT emission (i.e., $\mathscr{R}>10$ ), as shown by the arrows. Obviously the statistical significance of these data point is marginal, and a more quantita-

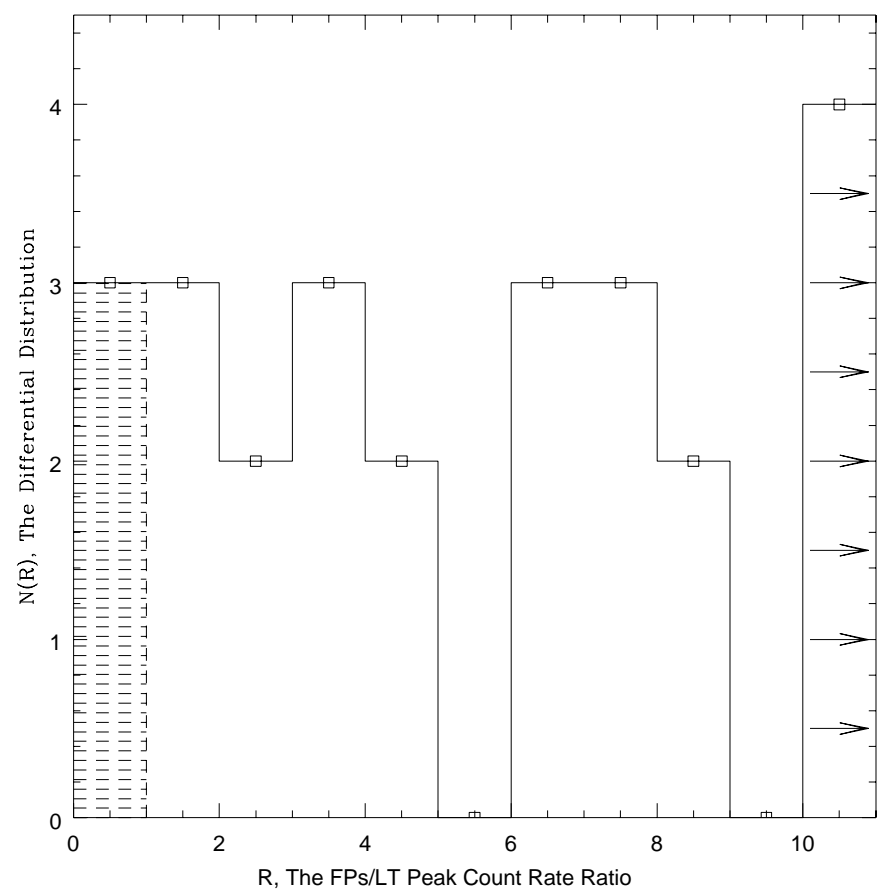

FIG. 11.-Differential distribution of the ratio $\mathscr{R}=$ FPs $/$ LT of the footpoints-to-loop top peak counts of all the flares for the entire mission (1991 October-1998 August). The arrows indicate ratios greater than the dynamic range of about 10 . Some of the flares in the shaded area may be occulted or be dominated by thermal emission from a superhot component. [See the electronic edition of the Journal for a color version of this figure.]

tive account of the relative distributions of the fluxes of the LT and FP sources requires a larger sample and dynamic range. Nevertheless, this kind of data is very important and can constrain the model parameters. As described in PD, this ratio can be related to the acceleration and the background plasma parameters. For example, it was shown that under certain conditions one has $\mathscr{R}=\tau_{\text {Coul }} / T_{\text {esc }}$, where $\tau_{\text {Coul }}$ and $T_{\text {esc }}$ are the Coulomb collision timescale in the loop and the escape time of the electrons from the acceleration site at the LT. However, for more general situations the relation of this ratio to the physical parameters is more complicated. As shown by numerical calculation in PD, values of a few for this ratio indicate plasma densities of about few times $10^{10} \mathrm{~cm}^{-3}$ and require a relatively long acceleration time compared to the escape time of the electrons from the LT source. Further constraints come from the consideration of the spectral shapes, which we discuss next.

\subsection{Spectral Indexes}

Another characteristic of the LT and FP emissions that can shed light on the acceleration mechanism is the relative shape of their spectra. The HXT instrument observes only at four broad channels, so that an accurate, absolute spectral analysis of individual sources is difficult and results are uncertain. However, the relative spectral characteristics of FP and LT sources are more reliable. To this end we have carried out the following analysis. We use the Yohkoh routine "hxtbox_fsp," which fits a specified model spectrum to the observed count rates and finds the best fit model parameters and calculates the $\chi^{2}$ values. When possible, we attempted to measure the spectral shape early in the impulsive phase (before or right at the first impulsive peak) so as to minimize any thermal contributions to the spectrum. 
For flare events with detectable images in the $\mathrm{H}$ band, we use the following four models:

1. a simple power law fitted to all four channels (Power Law 1);

2. a simple power law fitted to the three highest channels (Power Law 2), so that we avoid possible thermal contribution in the $\mathrm{L}$ band;

3. a broken power law with three parameters (low- and high-energy spectral indexes, $\gamma_{1}$ and $\gamma_{2}$, and the break energy, $E_{\mathrm{br}}$ ) fitted to all four channels (Broken Power Law $1)$; and

4. two simple power laws fitted separately to the $\mathrm{L}$ and M1 bands and to the M1 and M2 bands (Broken Power Law 2); in this case the break energy is fixed as the midpoint of the $\mathrm{M} 1$ band at $\sim 28 \mathrm{keV}$.

Table 2 summarizes these results.

We first note that in 14 out of 18 cases the value of the reduced $\chi^{2}, \chi^{2} / \nu$, is lowered when we remove the $\mathrm{L}$ band from the fit. The four-channel-fit $\chi^{2}$ values are lower, by statistically insignificant amounts, only for one FP and three LT sources. This combined with the results from the Broken Power Law 2 fits indicates that deviations from a power law is produced by the $\mathrm{L}$ band and that these could be in the form of a flattening or steepening of the spectrum with approximately equal probability. (The break energy $E_{\mathrm{br}}$ distribution, however, does not seem to agree with this conclusion. Only four out of nine values fall in or near the $\mathrm{L}$ band. This could be due to statistics of small sample.) The observed spectral change, of course, is not a reflection of the true behavior of the spectra because flares with detectable H-band flux must necessarily be biased toward those with flat and/or flattening spectra. Below we will compare the statistics of these flares with those without a detectable $\mathrm{H}$ band flux.
For flares with no usable images in the $\mathrm{H}$ band, we use two models: a simple power law fitted to the three channels (Power Law) and model 4 above (Broken Power Law 2). Table 3 presents the results of this analysis.

We first would like to note that the results from our analysis of the same events do not agree with Masuda's numbers. This could be either because the HXT grid response functions have been improved since Masuda did his analysis (see Sato, Kosugi, \& Makishima 1999) or due to the differences in the spatial boxes or time intervals used to calculate the indexes. Masuda does not specify these data. However, the general characteristics of the spectra (ranges, steepenings, etc.) of pre- and post-1993 September bursts given in the above table agree with the characteristics of 10 pre-1993 September spectra cited by Masuda.

Second, we note that in contrast to the sample of Table 2, only two sources (LT sources of 1992 February 17 and 1992 November 23) in Table 3 show a significant flattening. The rest maintain the power-law form or, in the majority of cases, steepen by a significant amount (a spectral index change of greater than 1), the spectral index changing by more than 3 in some sources. This difference between the flares from Tables 2 and 3 is due to the selection bias mentioned above and indicates that caution should be exercised in the interpretation of results from limited samples. The following figures describe the prominent features of the results from the whole sample.

Figure 12 shows the distribution of the spectral indexes for FP (solid histograms) and LT (dashed histograms) sources from the simple power law fits, for events with no $\mathrm{H}$ band images (left panel) and events with $\mathrm{H}$-band images (right panel). Several features are readily apparent. First, the main difference between the two samples is the absence of a significant number of high spectral index $(\gamma \gtrsim 6)$ events
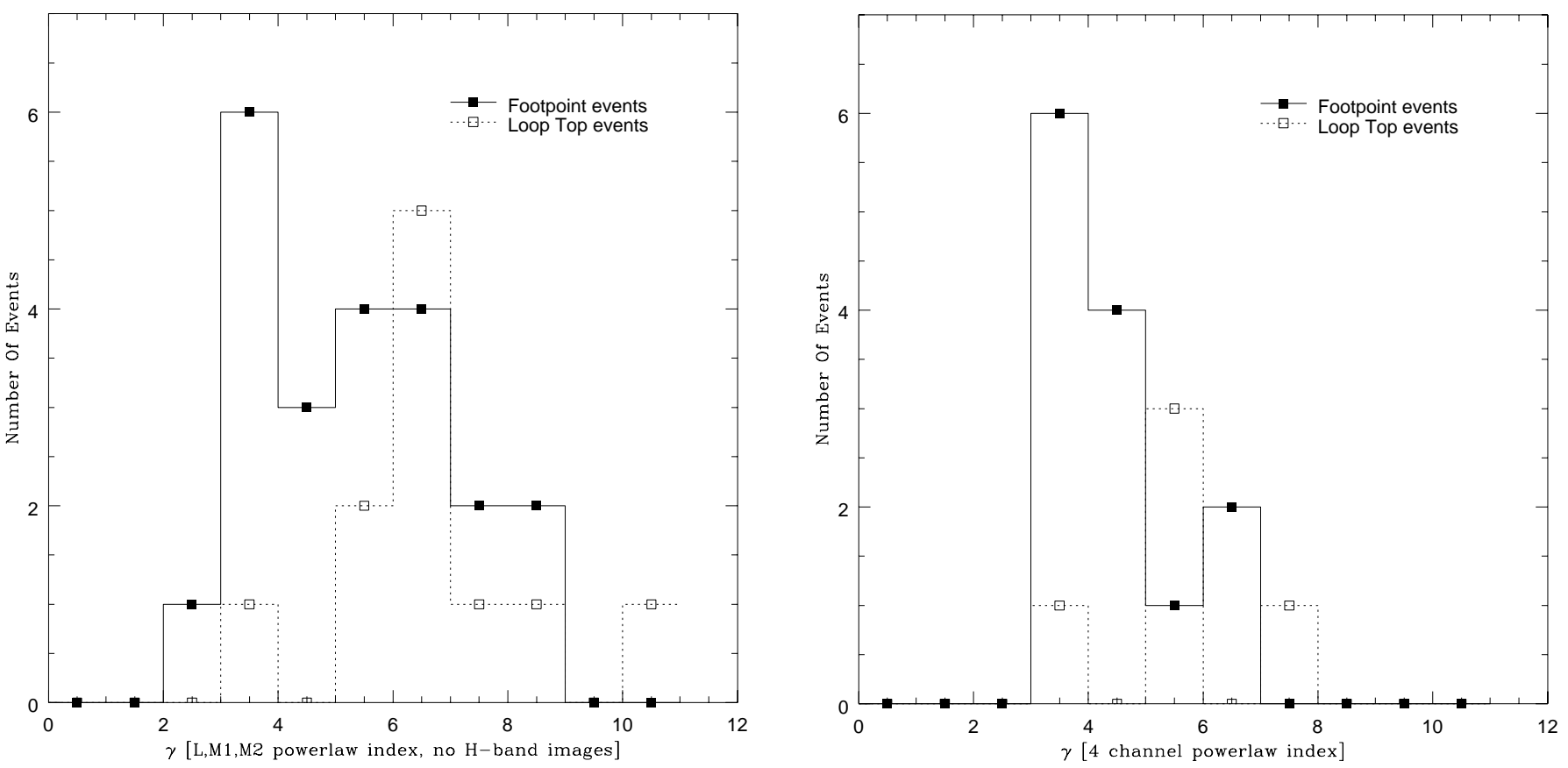

FIG. 12.-Distribution of power-law spectral indexes. Left panel: From fits to the L, M1, and M2 bands for all events with no detectable H-band images. Right panel: From fits to all four bands for events with detectable H-band images. The solid histograms and filled points represent the FP sources, and the dotted histogram and open points represent the loop top sources. [See the electronic edition of the Journal for a color version of this figure.] 

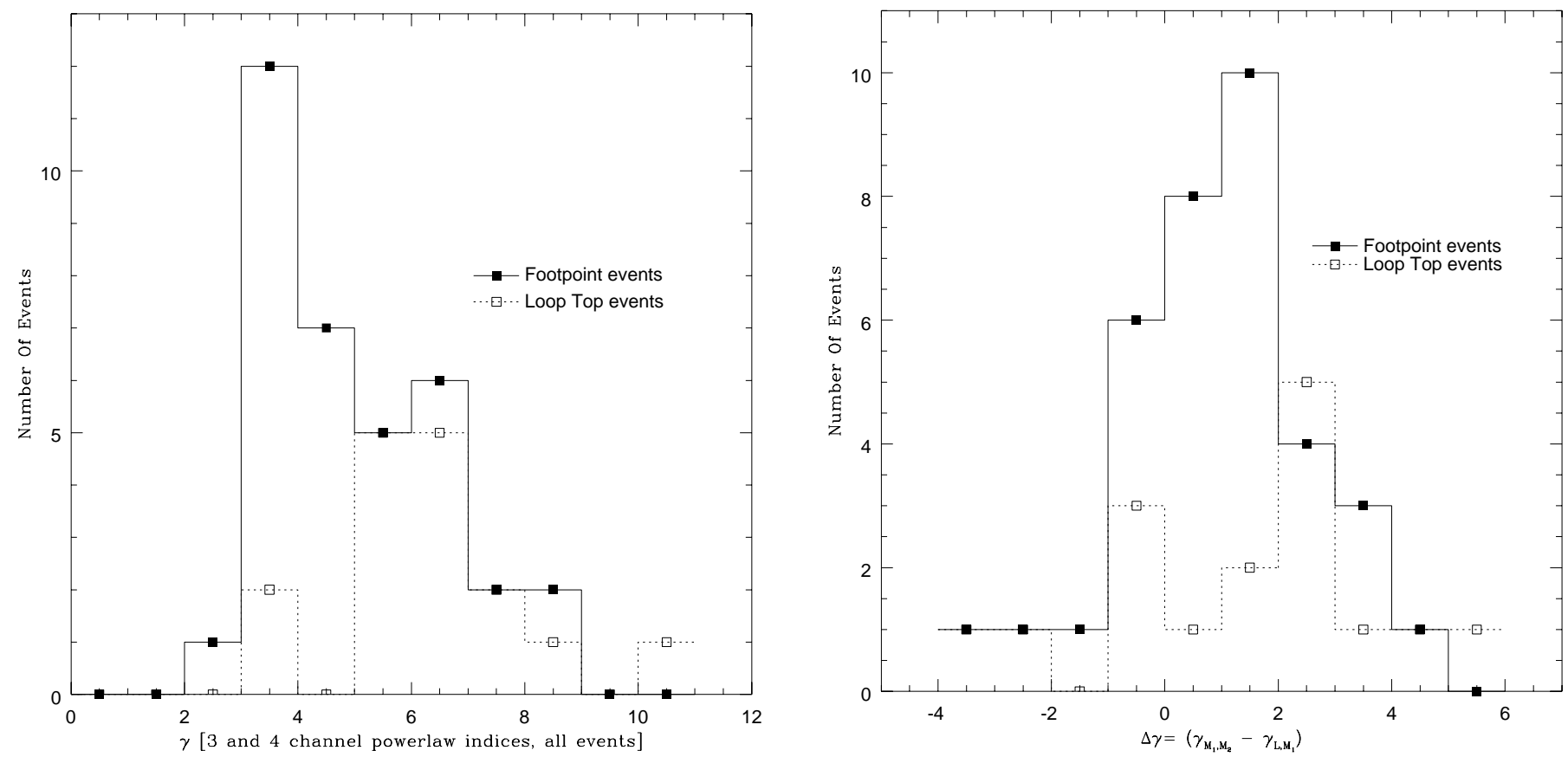

FIG. 13.-Distribution of the overall (three and four channel) index $\gamma$ (left panel) and the distribution of $\Delta \gamma=\gamma_{\mathrm{M} 1, \mathrm{M} 2}-\gamma_{\mathrm{L}, \mathrm{M} 1}$ for all 18 events (right panel). The solid histograms and filled points represent the FP sources, and the dotted histogram and open points represent the loop top sources. [See the electronic edition of the Journal for a color version of this figure.]

in the sample with H-band images (six vs. 16 in the sample with no H-band images) and a higher average spectral index, $\bar{\gamma}_{4 \mathrm{ch}}=4.65 \pm 1.2$ versus $\bar{\gamma}_{3 \mathrm{ch}}=5.73 \pm 1.5$. This is as expected. The HXT detects roughly equal counts in four channels from a flare with index $\gamma=4$, so that for $\gamma>6$ the counts in the $\mathrm{H}$ band will fall below that of the M2 band by a factor of 4 . This combined with our chosen threshold of 10 counts ${ }^{-1} \mathrm{SC}^{-1}$ for the $\mathrm{M} 2$ band indicates that such flares in our sample would have an H-band count rate of about 2-3 counts $\mathrm{s}^{-1} \mathrm{SC}^{-1}$ (which is at the 1 or $2 \sigma$ level) and will not yield a discernible image. (A background count rate of 2 counts $\mathrm{s}^{-1} \mathrm{SC}^{-1}$ for the $\mathrm{H}$ band implies $\sigma \sim 1$ or 2 counts $\mathrm{s}^{-1} \mathrm{SC}^{-1}$.) Thus, as stated above, this difference is purely due to the selection bias. In this connection we should note that spectral indexes larger than 6 or 7 based on minimum $\chi^{2}$ values may not be very reliable. Moreover, such sources may have a significant thermal contribution or be a superhot thermal source like the flare of 1992 February 6. If we ignore such flares (say those with $\gamma \geq 7.5$ ), then the two distributions become very similar to each other $\left(\bar{\gamma}_{4 \mathrm{ch}}=4.65 \pm 1.2\right.$ vs. $\left.\bar{\gamma}_{3 \mathrm{ch}}=5.03 \pm 1.3\right)$, and the overall distribution of the combined sample, which is shown in the left panel of Figure 13, becomes similar to previous determinations of this distribution (see McTiernan \& Petrosian 1991 and references therein; see also $\S 5$ ). Second, despite a sizable population of flares with $\gamma \simeq 3.0-4.0$, there exists a sharp cutoff at around $\gamma \simeq 3.0$ for both distributions. This must be intrinsic to the sources because there is no obvious selection bias that can produce it.

Finally, for both (and therefore the combined) samples, the LT sources tend to have larger indexes than the FP sources. This is evident in the above figures, which show a clear shift of the histogram of the LT sources relative to that of FPs (spectral index shift of about 1) and from the following average indexes: for three-channel, four-channel, and all sources, we get $\left(\bar{\gamma}_{\mathrm{LT}}=6.5 \pm 1.6, \bar{\gamma}_{\mathrm{FP}}=5.3 \pm 1.7\right)$,
$\left(\bar{\gamma}_{\mathrm{LT}}=5.5 \pm 1.3, \bar{\gamma}_{\mathrm{FP}}=4.3 \pm 1.0\right)$, and $\left(\bar{\gamma}_{\mathrm{LT}}=6.2 \pm 1.5\right.$, $\left.\bar{\gamma}_{\mathrm{FP}}=4.9 \pm 1.5\right)$, respectively.

Another important feature of the above results is the distribution of the spectral breaks. This effect is demonstrated by the right panel of Figure 13, where we show the distribution of $\Delta \gamma=\gamma_{\mathrm{M} 1, \mathrm{M} 2}-\gamma_{\mathrm{L}, \mathrm{M} 1}$ for all 18 events. The most noticeable trend in this figure is that, although there are both positive and negative values for $\Delta \gamma$, both the FP and LT distributions are weighted toward the positive side. The median values of $\Delta \gamma$ are about +1 and +2 for FP and LT sources, respectively. If we eliminate sources with $\gamma_{\mathrm{L}, \mathrm{M} 1}>6$, which may suffer from thermal contamination, the median values remain essentially unchanged but all sources with $\Delta \gamma \lesssim-0.5$ are eliminated. Considering the approximate nature of these values, the data are consistent with all thermally uncontaminated sources showing steepenings, i.e., $\Delta \gamma>0$. Higher resolution data extended to higher energies are required to clarify this picture.

As in the case of the flux ratios, the precision of our analysis is greatly limited by our small sample size and short spectral range coverage. Nonetheless, the above distributions provide us with initial data points that can be used to demonstrate how one can set constraints on the model parameters. As evident from the theoretical results presented in Figures 3, 5, and 9 of PD, spectral indexes $\gamma$ greater than 3 imply a short escape time in comparison to the acceleration time. This is similar to what we inferred from the distribution of the flux ratios and may favor the smaller ratio of pitch angle to momentum diffusion coefficients advocated in Pryadko \& Petrosian $(1997,1998,1999)$. The larger value of the spectral indexes of LT sources also tells us about the acceleration process. This difference in the spectral indexes of FP and LT sources is related, among other things, to the energy dependence of the pitch angle diffusion coefficient and/or the escape time. As shown by equation (15) in PD, $\gamma_{\mathrm{LT}}-\gamma_{\mathrm{FP}}=2-s / 2$, where $s$ is an index describing the 
energy dependence of the escape time (see eq. [5] in PD). Thus, the average value of about 1 for this difference seen in Figures 12 and 13 implies a value of $s=2$. Inspection of the above tables shows that the spectral difference for specific FP and LT sources associated with each other varies from 0 to 2 , indicating a wide range of 0 to +4 for $s$ in this simple parameterization. Clearly, these numbers cannot be taken too seriously but demonstrate how model parameters can be constrained using more refined data such as that expected from HESSI.

\section{CONCLUSIONS}

We have used the Yohkoh HXT Image Catalogue (Sato et al. 1998) to determine the frequency of occurrence of LT hard X-ray impulsive emission in solar flares and to compare the flux and spectral characteristics of these sources with those of the commonly observed FP sources. We have used Masuda's (1994) selection criteria and the Yohkoh spectral and spatial analyses software packages. In a few cases we have also used the Alexander \& Metcalf (1997) pixon method of image reconstruction. We determine the relative fluxes of the LT and FP sources and obtain rough measures for some of the spectral characteristics (e.g., power-law indexes) using all the possible ways of analysis that a four- or three-channel data set will allow. Analysis of these results lead us to the following conclusions:

1. The LT hard X-ray emission seems to be a common characteristic of the impulsive phase of solar flares, appearing in some form in 15 of the 18 selected flares. The absence of LT emission in the remaining cases is most likely due to the finite dynamic range of the imaging technique.

2. The ratio of the summed FP to LT fluxes lies in the range $10 \gtrsim \mathscr{R}=\mathrm{FPs} / \mathrm{LT} \gtrsim 1$ and has a relatively flat distribution in this range. The lower limit is intrinsic to the process, but the upper limit is most likely due to the finite (1 decade) dynamic range of the instrument and the imaging technique. Because of this it is difficult to know the true distribution of this ratio.

3. The overall distribution of the power-law spectral indexes rises rapidly above $\gamma=2$, peaks around 4 , and declines gradually thereafter. This is similar to previous determinations of this distribution from the Hard X-Ray Burst Spectrometer (HXRBS) on board the Solar Maximum Mission (McTiernan \& Petrosian 1991) but contains a few more steep spectra, specially for LT sources, which could be due to thermal contamination and lower energy range of HXT compared to HXRBS. This agreement is encouraging and indicates the reliability of our spectral determination.
4. The spectra tend to steepen at higher energies (spectral index $\gamma$ increases by 1 to 2), especially for sources with $\gamma<6$, for which the thermal contribution should be the lowest. This is the opposite of what is observed at higher energies, where spectra tend to flatten above hundreds of kilo-electron volts (McTiernan \& Petrosian 1991). The directivity of the X-ray emission and the albedo effect for the limb flares under consideration could also play some role, especially for the FP source (in the model discussed by PD, the LT source should emit isotropically). However, this is expected to be a small effect at the low energies $(<100 \mathrm{keV})$ under consideration here.

5. The spectral index $\gamma$ of LT sources is larger (i.e., spectra are steeper) than that of the FP sources on the average by $1\left(\bar{\gamma}_{\mathrm{LT}}=6.2 \pm 1.5, \bar{\gamma}_{\mathrm{FP}}=4.9 \pm 1.5\right)$. The differences between directivity and the albedo effects mentioned above could be a partial reason for such a behavior, but the physics of the acceleration process must certainly play a role here.

6. We have also described how the above results can be used to constrain the model parameters, especially those related to the acceleration process. For example, for models with acceleration at the LT source (see, e.g., Park, Petrosian, \& Schwartz 1997 and PD), the observed ranges of the flux ratios and spectral indexes indicate a rapid escape of the accelerated electron relative to the acceleration timescale, which is related to the momentum diffusion coefficient in the acceleration process. Moreover, the difference between spectral indexes of LT and FP sources can constrain the energy dependence of the escape time, which is related to the pitch angle diffusion rate in the acceleration site.

This demonstrates that similar studies of these characteristics of the flares can yield important information about the genesis and evolution of solar flares, and we eagerly anticipate the increased spectral, temporal, and spatial resolution possible with the instruments of the upcoming HESSI satellite.

Finally, we note that solar flares occur in many different morphologies, the most common being a simple flaring loop with one LT and two FP sources. However, as we discussed in $\S 3.2$, interacting loop models and even more complicated structures are frequently observed.

We would like to thank an anonymous referee for a careful reading of the original manuscript and for numerous helpful comments and suggestions that improved the paper considerably. This work is supported in part by NASA grants NAG5-7144-0002 and NAG5-8600-0001 and by a fellowship to T. Q. D. from Stanford's Undergraduate Research Opportunities.

\section{REFERENCES}

Alexander, D., \& Metcalf, T. R. 1997, ApJ, 489, 442

Aschwanden, M. J., Kosugi, T., Hanaoka, Y., Nishio, M., \& Melrose, D. 1999, ApJ, 526, 1026

Lee, T. T.,Petrosian, V., \& McTiernan, J. M. 1995, ApJ, 448, 915

Masuda, S. 1994, Ph.D. thesis, Univ. Tokyo

Masuda, S., Kosugi, T., Hara, H., Tsuneta, S., \& Ogawara, Y. 1994, Nature, 371, 495

McTiernan, J. M., \& Petrosian, V. 1991, ApJ, 379, 381

Park, B. T., Petrosian, V., \& Schwartz, R. A. 1997, ApJ, 489, 358

Petrosian, V. 1994, in AIP Conf. Proc. 294, High-Energy Solar Phenomena, ed. J. M. Ryan \& W. T. Vestrand (New York: AIP), 162

. 1996, in AIP Conf. Proc. 374, High Energy Solar Physics, ed. R.

Ramaty, N. Mandzhavidze, \& X.-M. Hua (Woodbury: AIP), 445
Petrosian, V., \& Donaghy, T. Q. 1999, ApJ, 527, 945

2000, in ASP Conf. Ser. 206, High Energy Solar Physics: Anticipating HESSI, ed. R. Ramaty \& N. Mandzhavidze (San Francisco: ASP), 215 (PD)

Pryadko, J., \& Petrosian, V. 1997, ApJ, 482, 774 1998, ApJ, 495, 377

1999 ApJ 515,873

Sato, J., Kosugi, T., \& Makishima, K. 1999, PASJ, 51, 127

Sato, J., Sawa, M., Masuda, S., Sakao, T., Kosugi, T., \& Sekiguchi, H. 1998, Yohkoh HXT Image Catalogue (Nagano: Noboyama Radio Obs.) 